\title{
SOLUTION OF THE TRUNCATED HYPERBOLIC MOMENT PROBLEM
}

\author{
RAÚL E. CURTO AND LAWRENCE A. FIALKOW
}

\begin{abstract}
Let $Q(x, y)=0$ be an hyperbola in the plane. Given real numbers $\beta \equiv \beta^{(2 n)}=\left\{\beta_{i j}\right\}_{i, j \geq 0, i+j \leq 2 n}$, with $\beta_{00}>0$, the truncated $Q$-hyperbolic moment problem for $\beta$ entails finding necessary and sufficient conditions for the existence of a positive Borel measure $\mu$, supported in $Q(x, y)=0$, such that $\beta_{i j}=\int y^{i} x^{j} d \mu \quad(0 \leq i+j \leq 2 n)$. We prove that $\beta$ admits a $Q$ representing measure $\mu$ (as above) if and only if the associated moment matrix $\mathcal{M}(n)(\beta)$ is positive semidefinite, recursively generated, has a column relation $Q(X, Y)=0$, and the algebraic variety $\mathcal{V}(\beta)$ associated to $\beta$ satisfies $\operatorname{card} \mathcal{V}(\beta) \geq \operatorname{rank} \mathcal{M}(n)(\beta)$. In this case, $\operatorname{rank} \mathcal{M}(n) \leq 2 n+1$; if $\operatorname{rank} \mathcal{M}(n) \leq 2 n$, then $\beta$ admits a rank $\mathcal{M}(n)$-atomic (minimal) $Q$-representing measure; if $\operatorname{rank} \mathcal{M}(n)=2 n+1$, then $\beta$ admits a $Q$-representing measure $\mu$ satisfying $2 n+1 \leq$ card $\operatorname{supp} \mu \leq 2 n+2$.
\end{abstract}

\section{IntRoduCtion}

Let $Q(x, y)=0$ denote an hyperbola in the plane. Given a real sequence $\beta \equiv \beta^{(2 n)}: \beta_{00}, \beta_{01}, \beta_{10}, \ldots, \beta_{0,2 n}, \beta_{2 n, 0}$, with $\beta_{00}>0$, we seek concrete necessary and sufficient conditions so that there exists a positive Borel measure $\mu$ on $\mathbb{R}^{2}$ satisfying

$$
\beta_{i j}=\int y^{i} x^{j} d \mu \quad(i, j \geq 0, i+j \leq 2 n)
$$

and

$$
\operatorname{supp} \mu \subseteq \mathcal{Z}(Q):=\left\{(x, y) \in \mathbb{R}^{2}: Q(x, y)=0\right\} ;
$$

a measure $\mu$ satisfying (1.1) is a representing measure for $\beta ; \mu$ is a $Q$-representing measure if it satisfies (1.1) and (1.2).

Our criterion for the existence of representing measures is expressed in terms of algebraic and geometric properties of the moment matrix $\mathcal{M}(n) \equiv \mathcal{M}(n)(\beta)$ that we next describe. The size of $\mathcal{M}(n)$ is $m(n):=(n+1)(n+2) / 2$, with rows and columns indexed as $1, X, Y, X^{2}, Y X, Y^{2}, \ldots, X^{n}, Y X^{n-1}, \ldots, Y^{n}$; the entry in row $Y^{i} X^{j}$, column $Y^{k} X^{\ell}$ of $\mathcal{M}(n)$ is $\beta_{i+k, j+\ell}$.

1991 Mathematics Subject Classification. Primary 47A57, 44A60, 42A70, 30A05; Secondary 15A57, 15-04, 47N40, 47A20.

Key words and phrases. Hyperbolic moment problem, moment matrix extension, flat extensions of positive matrices .

The first-named author's research was partially supported by NSF Research Grants DMS9800931 and DMS-0099357. The second-named author's research was partially supported by NSF Research Grant DMS-0201430. The second-named author was also partially supported by the State University of New York at New Paltz Research and Creative Projects Award Program. 
Let $\mathbb{R}_{n}[x, y]$ denote the space of real polynomials of degree at most $n$ in two variables, and let $\mathcal{B}_{n}$ denote the basis consisting of the monomials in degreelexicographic order, i.e., $\mathcal{B}_{n}: 1, x, y, x^{2}, y x, y^{2}, \ldots, x^{n}, y x^{n-1}, \ldots, y^{n}$. For $p \in \mathbb{R}_{2 n}[x, y]$, $p(x, y) \equiv \sum a_{r s} y^{r} x^{s}$, let $\hat{p}:=\left(a_{r s}\right)$ denote the coefficient vector of $p$ with respect to $\mathcal{B}_{2 n}$. Further, let $L_{\beta}: \mathbb{R}_{2 n}[x, y] \rightarrow \mathbb{R}$ be the Riesz functional defined by $L_{\beta}(p):=\sum a_{r s} \beta_{r s}$; then $\mathcal{M}(n)$ is uniquely determined by

$$
(\mathcal{M}(n) \hat{p}, \hat{q}):=L_{\beta}(p q) \quad\left(p, q \in \mathbb{R}_{n}[x, y]\right) .
$$

In particular, if $\mu$ is a representing measure for $\beta$, then $(\mathcal{M}(n) \hat{p}, \hat{p})=L_{\beta}\left(p^{2}\right)=$ $\int p^{2} d \mu \geq 0$. Since $\mathcal{M}(n)$ is real symmetric, it follows that $(\mathcal{M}(n)(\hat{p}+i \hat{q}), \hat{p}+i \hat{q}) \geq$ 0 , whence

$$
\mathcal{M}(n) \geq 0
$$

(i.e., $\mathcal{M}(n)$ is a positive semi-definite operator on $\left.\mathbb{C}^{m(n)}\right)$.

For $p \in \mathbb{R}_{n}[x, y], p(x, y) \equiv \sum a_{i j} y^{i} x^{j}$, we define an element $p(X, Y)$ of $\mathcal{C}_{\mathcal{M}(n)}$, the column space of $\mathcal{M}(n)$, by $p(X, Y):=\sum a_{i j} Y^{i} X^{j}$; for polynomials $p$ and $q$ with $\operatorname{deg} p+\operatorname{deg} q \leq n$, we also write $p(X, Y) q(X, Y)$ for $(p q)(X, Y)$. It follows from CuFi1, Proposition 3.1] that if $\mu$ is a representing measure for $\beta$, then

$$
\text { for } p \in \mathbb{R}_{n}[x, y], p(X, Y)=0 \Leftrightarrow \operatorname{supp} \mu \subseteq \mathcal{Z}(p) \text {. }
$$

It follows immediately from (1.5) that if $\beta$ has a representing measure, then $\mathcal{M}(n)$ is recursively generated in the following sense:

$$
p, q, p q \in \mathbb{R}_{n}[x, y], p(X, Y)=0 \Longrightarrow(p q)(X, Y)=0 .
$$

We define the variety of $\mathcal{M}(n)$ (or of $\beta$ ) by $\mathcal{V}(\mathcal{M}(n)):=\bigcap_{\substack{p \in \mathbb{R}_{n}[x, y] \\ p(X, Y)=0}} \mathcal{Z}(p)$; CuFi1, Proposition 3.1 and Corollary 3.7] implies that if $\mu$ is a representing measure for $\beta$, then $\operatorname{supp} \mu \subseteq \mathcal{V}(\mathcal{M}(n))$ and $\operatorname{rank} \mathcal{M}(n) \leq \operatorname{card} \operatorname{supp} \mu \leq \operatorname{card} \mathcal{V}(\mathcal{M}(n))$, whence $\mathcal{M}(n)$ satisfies the variety condition

$$
\operatorname{rank} \mathcal{M}(n) \leq \operatorname{card} \mathcal{V}(\mathcal{M}(n)) .
$$

In the sequel we repeatedly reply on the following basic result of CuFi1 Theorem 5.13]:

$\beta$ admits a $\operatorname{rank} \mathcal{M}(n)$-atomic (minimal) representing measure

if and only if $\mathcal{M}(n) \geq 0$ and $\mathcal{M}(n)$ admits an extension to

a (necessarily positive) moment matrix $\mathcal{M}(n+1)$ satisfying

$$
\operatorname{rank} \mathcal{M}(n+1)=\operatorname{rank} \mathcal{M}(n)
$$

we refer to such an extension as a flat extension.

Our main result shows that properties (1.4) - (1.7) completely characterize the existence of $Q$-representing measures, as follows.

Theorem 1.1. Let $Q(x, y)=0$ be an hyperbola in the plane. A sequence $\beta \equiv \beta^{(2 n)}$ has a representing measure supported in $Q(x, y)=0$ if and only if $\mathcal{M}(n)$ is positive semi-definite, recursively generated, $Q(X, Y)=0$ in $\mathcal{C}_{\mathcal{M}(n)}$, and $\operatorname{rank} \mathcal{M}(n) \leq$ card $\mathcal{V}(\mathcal{M}(n))$. In this case, rank $\mathcal{M}(n) \leq 2 n+1 ;$ if $\operatorname{rank} \mathcal{M}(n) \leq 2 n$, then there is a rank $\mathcal{M}(n)$-atomic $Q$-representing measure, while if rank $\mathcal{M}(n)=2 n+1$, there is a $Q$-representing measure $\mu$ for which $2 n+1 \leq$ card $\operatorname{supp} \mu \leq 2 n+2$. 
Consider the following property for a polynomial $P \in \mathbb{R}_{n}[x, y]$ :

$\left(A_{n}^{\prime}\right) \quad \beta \equiv \beta^{(2 n)}$ has a representing measure supported in $\mathcal{Z}(P)$ if and only if $\mathcal{M}(n)(\beta)$ is positive semi-definite, recursively generated, $P(X, Y)=0$ in $\mathcal{C}_{\mathcal{M}(n)}$, and $\operatorname{rank} \mathcal{M}(n) \leq \operatorname{card} \mathcal{V}(\mathcal{M}(n))$.

Polynomials which satisfy $\left(A_{n}^{\prime}\right)$ form an attractive class, because if $P$ satisfies $\left(A_{n}^{\prime}\right)$, then the degree- $2 n$ moment problem on $P(x, y)=0$ can be solved by concrete tests involving only elementary linear algebra and the calculation of roots of polynomials. Theorem 1.1 shows that each hyperbolic polynomial satisfies $\left(A_{n}^{\prime}\right)$ for $n \geq 2$. Moreover, $P$ satisfies $\left(A_{n}^{\prime}\right)$ for $n \geq \operatorname{deg} P$ if $P$ represents a line CuFi2, ellipse CuFi5, or parabola CuFi7. These results together yield the following.

Theorem 1.2. If $\operatorname{deg} P \leq 2$, then $P$ satisfies $\left(A_{n}^{\prime}\right)$ for every $n \geq \operatorname{deg} P$.

Despite Theorem 1.2 there are differences between the parabolic and elliptic moment problems and the hyperbolic problem. In the former cases, the conditions of $\left(A_{n}^{\prime}\right)$ always imply the existence of a $\operatorname{rank} \mathcal{M}(n)$-atomic representing measure, corresponding to a flat extension of $\mathcal{M}(n)$; for this reason, positive Borel measures supported on these curves always admit Gaussian cubature rules, i.e., rank $\mathcal{M}(n)$ atomic cubature rules of degree $2 n$ (cf. FiPe). By contrast, in the hyperbolic case, minimal representing measures $\mu$ sometimes entail card supp $\mu>\operatorname{rank} \mathcal{M}(n)$ (and Gaussian cubature rules may fail to exist; cf. Example [5.4).

The preceding results are part of a general study of truncated moment problems that we initiated in CuFi1, and are also related to the classical full moment problem, where moments of all orders are prescribed, i.e., $\beta \equiv \beta^{(\infty)}=\left(\beta_{i j}\right)_{i, j \geq 0}$ (cf. $\mathrm{AhKr}, \mathrm{Akh}, \mathrm{KrNu}, \mathrm{PuVa}, \mathrm{Schm1}$, ShTa, StSz2]). Theorem 1.2 is motivated in part by results of J. Stochel Sto1, who solved the full moment problem on planar curves of degree at most 2. Paraphrasing [Sto1] (i.e., translating from the language of moment sequences into the language of moment matrices), we consider the following property of a polynomial $P$ :

$$
\begin{aligned}
& \beta^{(\infty)} \text { has a representing measure supported in } P(x, y)=0 \\
& \text { if and only if } \mathcal{M}(\infty)(\beta) \geq 0 \text { and } P(X, Y)=0 \text { in } \mathcal{C}_{\mathcal{M}(\infty)} .
\end{aligned}
$$

Theorem 1.3. (Stochel [Sto1]) If $\operatorname{deg} P \leq 2$, then $P$ satisfies $(A)$.

In Sto1, Stochel also proved that there exist polynomials of degree 3 that do not satisfy $(A)$; Stochel and F. Szafraniec [StSz1] proved that there are polynomials of arbitrarily large degree that satisfy $(A)$ (cf. Fia3). Whether there exists a polynomial $P$ such that $P$ fails to satisfy $\left(A_{n}^{\prime}\right)$ for some $n \geq \operatorname{deg} P$ is an open problem (cf. Section [6).

The link between the truncated and full moment problems is provided by another result of Stochel (which actually holds for moment problems on $\mathbb{R}^{d}, d>1$ ).

Theorem 1.4. (cf. Sto2]) $\beta^{(\infty)}$ has a representing measure supported in a closed set $K \subseteq \mathbb{R}^{2}$ if and only if, for each $n, \beta^{(2 n)}$ has a representing measure supported in $K$.

In Section [6] we will use Theorem 1.4 to give a new proof of Theorem 1.3 To do so, we require the following refinement of Theorem 1.1] which relates the existence of representing measures to extensions of moment matrices. 
Theorem 1.5. Let $H:=\left\{(x, y) \in \mathbb{R}^{2}: Q(x, y)=0\right\}$ be an hyperbola. For $\beta \equiv \beta^{(2 n)}$, assume that $\mathcal{M}(n) \equiv \mathcal{M}(n)(\beta)$ is positive, recursively generated, and satisfies $Q(X, Y)=0$ in $\mathcal{C}_{\mathcal{M}(n)}$. Then rank $\mathcal{M}(n) \leq 2 n+1$, and the following statements are equivalent.

(i) $\beta$ admits a representing measure (necessarily supported in $H$ ).

(ii) $\beta$ admits a representing measure (necessarily supported in $H$ ) with convergent moments of degree up to $2 n+2$.

(iii) $\beta$ admits a representing measure $\mu$ (necessarily supported in $H$ ) satisfying card supp $\mu \leq 1+\operatorname{rank} M(n)$. If $\operatorname{rank} M(n) \leq 2 n$, then $\mu$ can be taken so that $\operatorname{card} \operatorname{supp} \mu=\operatorname{rank} M(n)$.

(iv) $M(n)$ admits a positive, recursively generated moment matrix extension $M(n+1)$.

(v) $M(n)$ admits a positive, recursively generated extension $M(n+1)$, with $\operatorname{rank} M(n+1) \leq 1+\operatorname{rank} M(n)$, and $M(n+1)$ admits a flat extension $M(n+2)$. If $\operatorname{rank} M(n) \leq 2 n$, then $M(n)$ admits a flat extension $M(n+1)$.

(vi) rank $M(n) \leq$ card $V(M(n))$.

Condition (vi) in Theorem 1.5] is the concrete condition which, together with positivity and recursiveness, provides an effective test for the existence of representing measures; we illustrate Theorem 1.5 with an example.

Example 1.6. We consider a case of $M(2)$ satisfying $Y X=1$; let

$$
\mathcal{M}(2)(\beta):=\left(\begin{array}{cccccc}
1 & 0 & 0 & a & 1 & a \\
0 & a & 1 & 0 & 0 & 0 \\
0 & 1 & a & 0 & 0 & 0 \\
a & 0 & 0 & c & a & 1 \\
1 & 0 & 0 & a & 1 & a \\
a & 0 & 0 & 1 & a & c
\end{array}\right)
$$

By calculating nested determinants, we see at once that the 3 by 3 upper left-hand corner is positive and invertible if and only if $a>1$, and that the 4 by 4 upper left-hand corner is positive and invertible if and only if $c>a^{2}$. We now let $a>1$ and $c:=a^{2}+r$, where $r>0$. Then $\operatorname{rank} M(2)=4$ if and only if

$$
\left(a^{2}-1\right)\left(r^{2}-a^{2}+1\right)=0 .
$$

The positive root of (1.9) is $r_{1}:=\sqrt{a^{2}-1}$, so we set $r:=r_{1}$ and observe that in $C_{\mathcal{M}(2)}, Y^{2}=2 a 1-X^{2}$. Since $Y X=1$, we next find the variety $V(\beta)$ by solving the pair of equations

$$
\left\{\begin{array}{c}
y^{2}=2 a-x^{2} \\
y=\frac{1}{x}
\end{array} .\right.
$$

It is easy to see that (1.10) has exactly four roots, $\left\{\left(x_{i}, y_{i}\right)\right\}_{i=1}^{4}$, where $x_{1}:=$ $-\left(a-\sqrt{a^{2}-1}\right), x_{2}:=a-\sqrt{a^{2}-1}, x_{3}:=-\left(a+\sqrt{a^{2}-1}\right), x_{4}:=a+\sqrt{a^{2}-1}$, and $y_{i}:=\frac{1}{x_{i}}(i=1,2,3,4)$. Thus, $\operatorname{rank} M(2)=4=\operatorname{card} V(M(2))$. According to Theorem 1.5 vi) $\Rightarrow(\mathrm{i}), \beta^{(4)}$ admits a representing measure $\mu$. Since $4=\operatorname{rank} M(2) \leq \operatorname{card} \operatorname{supp} \mu \leq \operatorname{card} V(\beta)=4$, it follows that $\operatorname{supp} \mu=V(\beta)$ and that $M(2)$ admits a flat extension $M(3)$ (cf. (1.8)).

Theorem 1.5 shows that minimal $Q$-representing measures for $\beta^{(2 n)}$ arise either from flat extensions of $\mathcal{M}(n)$ or of $\mathcal{M}(n+1)$ (cf. [Fia2]). In the presence of a flat 
extension, there is a simple procedure for computing the atoms and densities of a corresponding minimal representing measure.

Theorem 1.7. (cf. CuFi8, Theorem 2.21]) If $\mathcal{M}(n) \equiv \mathcal{M}(n)(\beta)$ is positive semi-definite and admits a flat extension $\mathcal{M}(n+1)$, then $\mathcal{V}:=\mathcal{V}(\mathcal{M}(n+1))$ satisfies card $\mathcal{V}=r(\equiv \operatorname{rank} \mathcal{M}(n))$, and $\mathcal{V} \equiv\left\{\left(x_{i}, y_{i}\right)\right\}_{i=1}^{r} \subseteq \mathbb{R}^{2}$ forms the support of the unique representing measure $\mu$ for $\mathcal{M}(n+1)$, i.e., $\mu$ is of the form $\mu=\sum_{i=1}^{r} \rho_{i} \delta_{\left(x_{i}, y_{i}\right)}$ with $\rho_{i}>0(1 \leq i \leq r)$. If $\mathcal{B} \equiv\left\{Y^{i_{k}} X^{j_{k}}\right\}_{k=1}^{r}$ is a maximal linearly independent subset of columns of $\mathcal{M}(n)$, let $V$ be the $r \times r$ matrix whose entry in row $k$, column $\ell$ is $y_{\ell}^{i_{k}} x_{\ell}^{j_{k}}(1 \leq k, \ell \leq r)$. Then $V$ is invertible, and $\rho \equiv\left(\rho_{1}, \ldots, \rho_{r}\right)$ is uniquely determined by $V \rho^{t}=\left(\beta_{i_{1}, j_{1}}, \ldots, \beta_{i_{r}, j_{r}}\right)^{t}$.

Example 1.8. (Example 1.6 cont.) We now use Theorem 1.7 to compute the densities for the measure $\mu:=\sum_{i=1}^{4} \rho_{i} \delta_{\left(x_{i}, y_{i}\right)}$ of Example 1.6 Since $\left\{1, X, Y, X^{2}\right\}$ is a basis for $\mathcal{C}_{\mathcal{M}(2)}$, Theorem 1.7 implies that

$$
V \equiv\left(\begin{array}{cccc}
1 & 1 & 1 & 1 \\
x_{1} & x_{2} & x_{3} & x_{4} \\
y_{1} & y_{2} & y_{3} & y_{4} \\
x_{1}^{2} & x_{2}^{2} & x_{3}^{2} & x_{4}^{2}
\end{array}\right),
$$

is invertible (indeed, $\left.\operatorname{det} V=\left(x_{1}^{2}-x_{3}^{2}\right)^{2}\right)$. The vector $\mathbf{v}:=\left(\beta_{i_{1}, j_{1}}, \ldots, \beta_{i_{r}, j_{r}}\right)^{t}$ in Theorem 1.7] is $(1,0,0, a)^{t}$, so a calculation of $\rho=V^{-1} \mathbf{v}$ shows that $\rho_{1}=\rho_{2}=\rho_{3}=$ $\rho_{4}=\frac{1}{4}$.

To prove Theorems 1.11 .2 and 1.5 we will reduce the analysis of truncated moment problems on conics to the study of truncated moment problems on four special conics: $x^{2}+y^{2}=1, y=x^{2}, y x=1$, and $y x=0$. This reduction was initially described (using complex moment matrices) in $\mathrm{CuFi6}$, but we now require a more detailed analysis.

For $a_{1}, a_{2}, b_{1}, b_{2}, c_{1}, c_{2} \in \mathbb{R}$, with $b_{1} c_{2} \neq b_{2} c_{1}$, let $\Phi: \mathbb{R}^{2} \rightarrow \mathbb{R}^{2}$ denote the degreeone bijection defined by $\Phi(x, y) \equiv\left(\varphi_{1}, \varphi_{2}\right):=\left(a_{1}+b_{1} x+c_{1} y, a_{2}+b_{2} x+c_{2} y\right)(x, y \in$ $\mathbb{R})$. Given $\beta^{(2 n)}$, define $\tilde{\beta}^{(2 n)}$ by $\tilde{\beta}_{i j}:=L_{\beta}\left(\varphi_{2}^{i} \varphi_{1}^{j}\right)(0 \leq i+j \leq 2 n)$, where $L_{\beta}$ denotes the Riesz functional associated with $\beta$. It is straightforward to verify that $L_{\tilde{\beta}}(p)=L_{\beta}(p \circ \Phi)$ for every $p \in \mathbb{R}_{n}[x, y]$. (Note that for $p(x, y) \equiv \sum a_{i j} y^{i} x^{j}$, $(p \circ \Phi)(x, y)=p\left(\varphi_{1}, \varphi_{2}\right) \equiv \sum a_{i j} \varphi_{2}^{i} \varphi_{1}^{j}$.) Also, $L_{\beta}(p)=L_{\tilde{\beta}}(p \circ \Psi)$, where $\Psi:=$ $\Phi^{-1}$, given by $\Psi(u, v):=\frac{1}{b_{1} c_{2}-b_{2} c_{1}}\left(c_{2}\left(u-a_{1}\right)-c_{1}\left(v-a_{2}\right),-b_{2}\left(u-a_{1}\right)+b_{1}\left(v-a_{2}\right)\right)$.

Let $\mathcal{H}^{(n)}:=\left\{\hat{p}: p \in \mathbb{R}_{n}[x, y]\right\} \cong \mathbb{R}^{m(n)}$ and consider the linear map $J \equiv J^{(n)}$ : $\mathcal{H}^{(n)} \rightarrow \mathcal{H}^{(n)}$ defined by $J(\hat{p}):=\widehat{p \circ \Phi}\left(p \in \mathbb{R}_{n}[x, y]\right)$. The map $J$ is invertible, with inverse $J^{-1}(\hat{q}):=\widehat{q \circ \Psi}\left(q \in \mathbb{R}_{n}[x, y]\right)$. Note that $\mathcal{H}^{(n)}$ admits a vector space direct sum decomposition $\mathcal{H}^{(n)}=\mathcal{H}^{(n-1)} \bigoplus \mathcal{H}_{n}$, where $\mathcal{H}_{n}$ is the subspace spanned by vectors $\widehat{y^{i} x^{j}}$ with $i+j=n$. Since $J^{(n)}$ and $J^{(n-1)}: \mathcal{H}^{(n-1)} \rightarrow \mathcal{H}^{(n-1)}$ are both invertible, relative to the above decomposition $J^{(n)}$ can be represented as

$$
J^{(n)}=\left(\begin{array}{cc}
J^{(n-1)} & K_{n} \\
0 & L_{n}
\end{array}\right),
$$

and thus $\left(J^{(n)}\right)^{-1}$ admits a similar triangular representation.

Proposition 1.9. (Invariance under degree-one transformations.) Let $\mathcal{M}(n)$ and $\tilde{\mathcal{M}}(n)$ be the moment matrices associated with $\beta$ and $\tilde{\beta}$.

(i) $\tilde{\mathcal{M}}(n)=J^{*} \mathcal{M}(n) J$ 
(ii) $J$ is invertible.

(iii) $\tilde{\mathcal{M}}(n) \geq 0 \Leftrightarrow \mathcal{M}(n) \geq 0$.

(iv) $\operatorname{rank} \tilde{\mathcal{M}}(n)=\operatorname{rank} \mathcal{M}(n)$.

(v) The formulas $\mu=\tilde{\mu} \circ \Phi$ and $\tilde{\mu}=\mu \circ \Psi$ establish a one-to-one correspondence between the sets of representing measures for $\beta$ and $\tilde{\beta}$, which preserves measure class and cardinality of the support; moreover, $\Phi(\operatorname{supp} \mu)=\operatorname{supp} \tilde{\mu}$ and $\Psi(\operatorname{supp} \tilde{\mu})=\operatorname{supp} \mu$.

(vi) For $p \in \mathbb{R}_{n}[x, y], p(\tilde{X}, \tilde{Y})=J^{*}((p \circ \Phi)(X, Y))$ and $p(X, Y)=$ $\left(J^{-1}\right)^{*}((p \circ \Psi)(\tilde{X}, \tilde{Y}))$.

(vii) $\tilde{\mathcal{M}}(n)$ is recursively generated if and only if $\mathcal{M}(n)$ is recursively generated.

(viii) $\Phi(\mathcal{V}(\beta))=\mathcal{V}(\tilde{\beta})$ and $\mathcal{V}(\beta)=\Psi(\mathcal{V}(\tilde{\beta}))$

(ix) $\mathcal{M}(n)$ is positive semi-definite and admits a positive, recursively generated (resp. flat) extension $\mathcal{M}(n+1)$ if and only if $\tilde{\mathcal{M}}(n)$ is positive semi-definite and admits a positive, recursively generated (resp. flat) extension $\tilde{\mathcal{M}}(n+1)$.

Proof. We omit the proofs of (i) - (viii), which are straightforward. For (ix), suppose $\mathcal{M}(n)$ is positive and admits a positive, recursively generated extension

$$
\mathcal{M}(n+1)(\check{\beta}) \equiv\left(\begin{array}{cc}
\mathcal{M}(n) & B(n+1) \\
B(n+1)^{t} & C(n+1)
\end{array}\right) .
$$

Part (i) (using $n+1$ instead of $n$ ) and (1.11) imply that

$$
\begin{aligned}
\mathcal{M} & \equiv \tilde{\mathcal{M}}(n+1)=\left(J^{(n+1)}\right)^{*} \mathcal{M}(n+1)(\check{\beta}) J^{(n+1)} \\
& =\left(\begin{array}{cc}
\left(J^{(n)}\right)^{*} \mathcal{M}(n) J^{(n)} & * \\
* & *
\end{array}\right)=\left(\begin{array}{cc}
\tilde{\mathcal{M}}(n) & * \\
* & *
\end{array}\right) .
\end{aligned}
$$

Thus, $\mathcal{M}$ is a positive moment matrix extension of $\tilde{\mathcal{M}}(n)$, and (vii) (applied with $n+1)$ implies that $\mathcal{M}$ is recursively generated. In the case when $\mathcal{M}$ is a flat extension, we have, from (i), (ii) and (iv), $\operatorname{rank} \tilde{\mathcal{M}}(n)=\operatorname{rank} \mathcal{M}(n)=\operatorname{rank} \mathcal{M}(n+$ 1) $(\check{\beta})=\operatorname{rank} \mathcal{M}$, so $\mathcal{M}$ is a flat extension of $\tilde{\mathcal{M}}(n)$. The converse is proved in the same way.

It is well known that a general conic $Q(x, y)=0$ can be transformed into one of the four special cases by means of rotation, scaling and translation; thus, there is a degree-one map $\Phi$ (as above) such that $Q \circ \Phi$ is a special conic. Note from Proposition 1.9 that such properties of $\mathcal{M}(n)$ as positivity, recursiveness, the variety condition, and the existence of flat or positive extensions $\mathcal{M}(n+1)$ are invariant under degree-one mappings, which also preserve the existence of representing measures and the cardinality of the support. These observations show that Theorems 1.1 and 1.5 are valid for arbitrary hyperbolas if and only if they are valid for $y x=1$ and $y x=0$. We prove Theorem 1.1 for $y x=1$ in Section 2 (Theorem 2.1) and for $y x=0$ in Section 3 (Theorem [3.1). We prove Theorem 1.5 (for both hyperbolas) in Section 4 (Theorem 4.1). Section 5 contains examples illustrating our results. Section [6] contains a new proof of Theorem 1.3 based on moment matrix techniques, including Theorem [1.5]

The remainder of this section is devoted to notation and basic results concerning real moment matrices. Given a collection $\beta^{(2 n)}: \beta_{00}, \beta_{01}, \beta_{10}, \ldots, \beta_{0,2 n}, \ldots, \beta_{2 n, 0}$, we can describe $\mathcal{M}(n)(\beta)$ by means of a block matrix decomposition $\mathcal{M}(n)(\beta):=$ 
$\left(\mathcal{M}_{i j}(\beta)\right)_{i, j=0}^{n}$, where

$$
\mathcal{M}_{i j}(\beta):=\left(\begin{array}{cccc}
\beta_{0, i+j} & \beta_{1, i+j-1} & \cdots & \beta_{j, i} \\
\beta_{1, i+j-1} & \beta_{2, i+j-2} & \cdots & \beta_{j+1, i-1} \\
\vdots & \vdots & \ddots & \vdots \\
\beta_{i, j} & \beta_{i+1, j-1} & \cdots & \beta_{i+j, 0}
\end{array}\right) .
$$

Recall that $\mathcal{M}(n)$ has size $m(n) \equiv \frac{(n+1)(n+2)}{2}$. For any matrix $M$ of this size, $[M]_{k}$ denotes the compression of $M$ to the first $k$ rows and columns; similarly, for a vector $\mathbf{v},[\mathbf{v}]_{k}$ denotes the compression of $\mathbf{v}$ to the first $k$ entries. We also consider compressions of $M$ and $\mathbf{v}$ to a set $E$ of rows and columns, and denote such compressions by $[M]_{E}$ and $[\mathbf{v}]_{E}$. For $i+j, k+\ell \leq n,\left\langle Y^{i} X^{j}, Y^{k} X^{l}\right\rangle_{M}$ (or simply $\left\langle Y^{i} X^{j}, Y^{k} X^{l}\right\rangle$ ) denotes the entry of $M$ in row $Y^{k} X^{l}$ and column $Y^{i} X^{j}$.

A theorem of Smul'jan Smu shows that a block matrix

$$
M=\left(\begin{array}{cc}
A & B \\
B^{*} & C
\end{array}\right)
$$

is positive if and only if (i) $A \geq 0$, (ii) there exists a matrix $W$ such that $B=A W$, and (iii) $C \geq W^{*} A W$ (since $A=A^{*}, W^{*} A W$ is independent of $W$ satisfying $B=A W)$. Note also that if $M \geq 0$, then $\operatorname{rank} M=\operatorname{rank} A$ if and only if $C=W^{*} A W$; conversely, if $A \geq 0$ and there exists $W$ such that $B=A W$ and $C=W^{*} A W$, then $M \geq 0$ and $\operatorname{rank} M=\operatorname{rank} A$. In the sequel, for $A \geq 0$, we refer to $M$ as an extension of $A$, and as a flat extension if $\operatorname{rank} M=\operatorname{rank} A$. Thus, a flat extension of a positive matrix $A$ is completely determined by a choice of block $B$ satisfying $B=A W$ and $C=W^{*} A W$ for some matrix $W$; we denote such an extension by $[A ; B]$. It follows from the Extension Principle Fia1 that if $M \geq 0$, then column dependence relations in $A$ extend to $\left(\begin{array}{c}A \\ B^{*}\end{array}\right)$; further, if $M$ is a flat extension of $A(\geq 0)$, then column dependence relations in $\left(\begin{array}{ll}A & B\end{array}\right)$ extend to $M$.

For an $(n+1) \times(n+2)$ moment matrix block $B_{n, n+1}$, representing "new moments" of degree $2 n+1$ for a prospective representing measure of $\beta^{(2 n)}$, let

$$
B(n+1):=\left(\begin{array}{c}
B_{0, n+1} \\
\vdots \\
B_{n-1, n+1} \\
B_{n, n+1}
\end{array}\right)
$$

By Smul'jan's theorem, $\mathcal{M}(n) \geq 0$ admits a (necessarily positive) flat extension

$$
[\mathcal{M}(n) ; B]=\left(\begin{array}{cc}
\mathcal{M}(n) & B \\
B^{*} & C
\end{array}\right)
$$

in the form of a moment matrix $\mathcal{M}(n+1)$ if and only if

$$
\begin{aligned}
& B=B(n+1) \text { and } B=\mathcal{M}(n) W \text { for some } W \\
& \text { (i.e., } \operatorname{Ran} B \subseteq \operatorname{Ran} \mathcal{M}(n)[\text { Dou }] \text { ); and } \\
& C:=W^{*} \mathcal{M}(n) W \text { is Hankel } \\
& \text { (i.e., } \left.C \text { has the form of a moment matrix block } B_{n+1, n+1}\right) \text {. }
\end{aligned}
$$

Acknowledgement. The examples in this paper were obtained using calculations with the software tool Mathematica Wol. 


\section{The Truncated Moment Problem on Nondegenerate Hyperbolas}

In this section we prove Theorem 1.1 for nondegenerate hyperbolas. In view of Proposition [1.9] and the remarks following it, it suffices to consider the case $y x=1$. The necessity of the conditions in Theorem 1.1 is clear from Section 1 . and sufficiency follows immediately from Theorem 2.1 below .

Theorem 2.1. Let $\beta \equiv \beta^{(2 n)}: \beta_{00}, \beta_{01}, \beta_{10}, \ldots, \beta_{0,2 n}, \ldots, \beta_{2 n, 0}$ be a family of real numbers, $\beta_{00}>0$, and let $\mathcal{M}(n)$ be the associated moment matrix. Assume that $\mathcal{M}(n)$ is positive, recursively generated, and satisfies $Y X=1$ and rank $\mathcal{M}(n) \leq$ card $\mathcal{V}(\beta)$. Then $\operatorname{rank} \mathcal{M}(n) \leq 2 n+1$. If $\operatorname{rank} \mathcal{M}(n) \leq 2 n$, then $\mathcal{M}(n)$ admits a flat extension $\mathcal{M}(n+1)$ (so $\beta$ admits a rank $\mathcal{M}(n)$-atomic representing measure supported in $y x=1)$. If rank $\mathcal{M}(n)=2 n+1$, then $\mathcal{M}(n)$ admits an extension to a positive, recursively generated extension $\mathcal{M}(n+1)$, satisfying $2 n+1 \leq \operatorname{rank} \mathcal{M}(n+$ $1) \leq 2 n+2$, and $\mathcal{M}(n+1)$ admits a flat extension $\mathcal{M}(n+2)$ (so $\beta$ admits a representing measure $\mu$ supported in $y x=1$, with $2 n+1 \leq$ card $\operatorname{supp} \mu \leq 2 n+2$.

We require several preliminary results for the proof of Theorem 2.1] By CuFi2, Theorem 2.1], we know that $\mathcal{M}(n)$ (positive semi-definite and recursively generated) admits flat extensions when $\{1, X, Y\}$ is linearly dependent in $\mathcal{C}_{\mathcal{M}(n)}$. Thus, hereafter we will assume that $\{1, X, Y\}$ is linearly independent. We begin with an elementary lemma that exploits the fact that $\mathcal{M}(n)$ is recursively generated. For $1 \leq k \leq n$ let

$$
\mathcal{S}_{n}(k):=\left\{1, X, Y, X^{2}, Y^{2}, \ldots, X^{k}, Y^{k}\right\} \subseteq \mathcal{C}_{\mathcal{M}(n)} .
$$

Lemma 2.2. For $n \geq 2$, let $\mathcal{M}(n)$ be positive and recursively generated, and assume that $Y X=1$. Then each column of $\mathcal{M}(n)$ is equal to a column in $\mathcal{S}_{n}(n)$; in particular, $\operatorname{rank} \mathcal{M}(n) \leq 2 n+1$.

Proof. The proof is by induction on $n \geq 2$. For $n=2$ the statement is clearly true, so assume it holds for $n=k(\geq 2)$. Suppose $\mathcal{M}(k+1)$ is positive and recursively generated, with $Y X=1$ in $\mathcal{C}_{\mathcal{M}(k+1)}$. Let $i, j \geq 0, i+j \leq k$. By the induction hypothesis, each column of the form $\left[Y^{i} X^{j}\right]_{m(k)}$ is in $\mathcal{S}_{k}(k)$, and since $\mathcal{M}(k+1) \geq 0$, the Extension Principle [Fia1 Proposition 2.4] shows that $Y^{i} X^{j} \in \mathcal{S}_{k}(k+1)\left(\subseteq \mathcal{S}_{k+1}(k+1)\right)$. Since $X^{k+1}, Y^{k+1} \in \mathcal{S}_{k+1}(k+1)$, it now suffices to consider a column in $\mathcal{M}(k+1)$ of the form $Y^{k+1-j} X^{j}$, with $1 \leq j \leq k$. Let $q(x, y):=y x-1$ and let $p_{i j}(x, y):=y^{i} x^{j}$, so that $Y^{k+1-j} X^{j}=p_{k+1-j, j}(X, Y)$. Also, let $r_{i j}(x, y):=y^{k+1-j} x^{j}-y^{k-j} x^{j-1}$. Now $r_{i j}(x, y)=y^{k-j} x^{j-1}(y x-1)=$ $p_{k-j, j-1}(x, y) q(x, y)$; since $\mathcal{M}(k+1)$ is recursively generated and $q(X, Y)=0$, it follows that $r_{i j}(X, Y)=0$, that is, $Y^{k+1-j} X^{j}=Y^{k-j} X^{j-1}$ in $\mathcal{C}_{\mathcal{M}(k+1)}$. By induction, $\left[Y^{k-j} X^{j-1}\right]_{m(k)} \in \mathcal{S}_{k}(k)$, and since $\mathcal{M}(k+1) \geq 0$, it follows as above that $Y^{k-j} X^{j-1} \in \mathcal{S}_{k+1}(k)$. Thus $Y^{k+1-j} X^{j}\left(=Y^{k-j} X^{j-1}\right) \in \mathcal{S}_{k+1}(k) \subseteq \mathcal{S}_{k+1}(k+$ 1), as desired.

We next present two auxiliary results that will be used frequently in the sequel. Recall that for $i+j, k+\ell \leq n,\left\langle Y^{i} X^{j}, Y^{k} X^{\ell}\right\rangle$ denotes the entry of $\mathcal{M}(n)$ in row $Y^{k} X^{\ell}$, column $Y^{i} X^{j}$, namely $\beta_{i+k, j+\ell}$. We extend this inner product notation from monomials to polynomials as follows. For $p \equiv \sum_{0 \leq i+j \leq n} a_{i j} y^{i} x^{j}$ and $q \equiv$ $\sum_{0 \leq k+\ell \leq n} b_{k \ell} y^{k} x^{\ell}$, we define $\langle p(X, Y), q(X, Y)\rangle:=\sum_{0 \leq i+j, k+\ell \leq n} a_{i j} b_{k \ell} \beta_{i+k, j+\ell}$. Further, if $\operatorname{deg} p+\operatorname{deg} p^{\prime}, \operatorname{deg} q+\operatorname{deg} q^{\prime} \leq n, \operatorname{by}\left\langle p(X, Y) p^{\prime}(X, Y), q(X, Y) q^{\prime}(X, Y)\right\rangle$ we mean $\left\langle\left(p p^{\prime}\right)(X, Y),\left(q q^{\prime}\right)(X, Y)\right\rangle$. The following result follows directly from the preceding definitions. 
Lemma 2.3. (i) For $p, q \in \mathbb{R}_{n}[x, y]$,

$$
\langle p(X, Y), q(X, Y)\rangle=\langle q(X, Y), p(X, Y)\rangle .
$$

(ii) For $p, q \in \mathbb{R}_{n}[x, y], i, j \geq 0, i+j \leq n$, and $\operatorname{deg} p, \operatorname{deg} q \leq n-(i+j)$,

$$
\left\langle p(X, Y) Y^{j} X^{i}, q(X, Y)\right\rangle=\left\langle p(X, Y), q(X, Y) Y^{j} X^{i}\right\rangle .
$$

(iii) If $p, q, r \in \mathbb{R}_{n}[x, y]$ with $p(X, Y)=q(X, Y)$ in $\mathcal{C}_{\mathcal{M}(n)}$, then $\langle r(X, Y), p(X, Y)\rangle$ $=\langle r(X, Y), q(X, Y)\rangle$.

Lemma 2.4. Let $\mathcal{M}(n)$ be positive, recursively generated, with $Y X=1$, and assume $p, q \in \mathbb{R}_{n-1}[x, y]$. Then

$$
\begin{aligned}
\langle p(X, Y), q(X, Y)\rangle & =\langle Y p(X, Y), X q(X, Y)\rangle \\
& =\langle X p(X, Y), Y q(X, Y)\rangle .
\end{aligned}
$$

Proof. The definition of $\langle p(X, Y), q(X, Y)\rangle$ implies that, without loss of generality, we can assume that $p(X, Y)=Y^{i} X^{j}$ and $q(X, Y)=Y^{k} X^{\ell}$. Assume first that $k \geq 1$. We have

$$
\begin{aligned}
\left\langle Y^{i} X^{j}, Y^{k} X^{\ell}\right\rangle & =\left\langle Y^{i+1} X^{j}, Y^{k-1} X^{\ell}\right\rangle \quad \text { (by Lemma2.3(ii)) } \\
& =\left\langle Y^{i+1} X^{j}, Y^{k-1} X^{\ell} Y X\right\rangle \quad \text { (by Lemma[2.3(iii), using } Y X=1 \text { ) } \\
& =\left\langle Y^{i+1} X^{j}, Y^{k} X^{\ell+1}\right\rangle .
\end{aligned}
$$

If $k=0$ and $j \geq 1$, we have

$$
\begin{aligned}
\left\langle Y^{i} X^{j}, X^{\ell}\right\rangle & =\left\langle Y^{i} X^{j-1}, X^{\ell+1}\right\rangle \quad \text { (by Lemma 2.3(ii)) } \\
& \left.=\left\langle Y^{i} X^{j-1} Y X, X^{\ell+1}\right\rangle \quad \text { (by Lemma2.3(iii), using } Y X=1\right) \\
& =\left\langle Y^{i+1} X^{j}, X^{\ell+1}\right\rangle .
\end{aligned}
$$

If $k=j=0$, we have $p(X, Y)=Y^{i}, q(X, Y)=X^{\ell}$, so we need to prove that $\left\langle Y^{i}, X^{\ell}\right\rangle=\left\langle Y^{i+1}, X^{\ell+1}\right\rangle$. If $i \geq 1$, we have

$$
\begin{aligned}
\left\langle Y^{i}, X^{\ell}\right\rangle & =\left\langle Y^{i-1}, Y X^{\ell}\right\rangle \quad \text { (by Lemma 2.3(ii)) } \\
& =\left\langle Y^{i} X, Y X^{\ell}\right\rangle \quad \text { (by Lemma[2.3(i),(iii)) } \\
& =\beta_{i+1, \ell+1}=\left\langle Y^{i+1}, X^{\ell+1}\right\rangle .
\end{aligned}
$$

If $i=0$, then

$$
\begin{gathered}
\left\langle Y^{i}, X^{\ell}\right\rangle=\left\langle 1, X^{\ell}\right\rangle=\left\langle Y X, X^{\ell}\right\rangle \quad \text { (by Lemma[2.3(iii)) } \\
\left\langle Y, X^{\ell+1}\right\rangle \quad \text { (by Lemma[2.3(ii)). }
\end{gathered}
$$

We have now completed the proof of (2.1); the validity of (2.2) is a straightforward consequence of (2.1) and Lemma 2.3(i).

We next divide the proof of Theorem 2.1 into four cases, based on possible dependence relations among the elements of $\mathcal{S}_{n}(n)$. Section 5 contains examples illustrating these cases. In proving each case, we ultimately obtain some flat moment matrix extension $\mathcal{M}$; the existence of a corresponding $\operatorname{rank} \mathcal{M}$-atomic representing measure $\mu$ supported in $y x=1$ then follows immediately from (1.8) and (1.5); for this reason, and to simplify the statement of each case, we address only the matrix extension, not the representing measure. In the sequel, unless otherwise noted, 
we are always assuming that $\mathcal{M}(n)$ is positive, recursively generated, $\{1, X, Y\}$ is linearly independent, $Y X=1$, and $\operatorname{rank} \mathcal{M}(n) \leq \operatorname{card} \mathcal{V}(\beta)$.

Proposition 2.5. (Case I: For some $k, 2 \leq k \leq n, \mathcal{S}_{n}(k-1)$ is linearly independent and $X^{k} \in$ lin.span $\mathcal{S}_{n}(k-1)$ ) Assume that $\mathcal{M}(n)(\beta)$ is positive, recursively generated, satisfies $Y X=1$, and $\operatorname{rank} \mathcal{M}(n) \leq \operatorname{card} \mathcal{V}(\beta)$. In $\mathcal{S}_{n}(n)$, assume that the first dependence relation occurs at $X^{k}$, with $2 \leq k \leq n$. Then $\mathcal{M}(n)$ is flat and, a fortiori, it admits a unique flat extension $\mathcal{M}(n+1)$.

Proof. Write $X^{k}=p_{k-1}(X)+q_{k-1}(Y)$, where $\operatorname{deg} p_{k-1}, \operatorname{deg} q_{k-1} \leq k-1$. It follows that $\mathcal{V}(\beta) \subseteq(y x=1) \bigcap\left(p_{k-1}(x)+q_{k-1}(y)=x^{k}\right) \subseteq(y x=1) \bigcap\left(p_{k-1}(x)+q_{k-1}\left(\frac{1}{x}\right)=\right.$ $\left.x^{k}\right)$. Since $p_{k-1}(x)+q_{k-1}\left(\frac{1}{x}\right)=x^{k}$ leads to a polynomial equation in $x$ of degree at most $2 k-1$, it follows that card $\mathcal{V}(\beta) \leq 2 k-1$, so $\operatorname{rank} \mathcal{M}(n) \leq 2 k-1$. Then $\mathcal{S}_{n}(k-1) \equiv\left\{1, X, Y, X^{2}, Y^{2}, \ldots, X^{k-1}, Y^{k-1}\right\}$ is a basis for $\mathcal{C}_{\mathcal{M}(n)}$, whence $\mathcal{M}(n)$ is flat.

Proposition 2.6. (Case II: For some $k, 2 \leq k<n, \mathcal{S}_{n}(k-1) \bigcup\left\{X^{k}\right\}$ is linearly independent, and $Y^{k} \in$ lin.span $\left(\mathcal{S}_{n}(k-1) \bigcup\left\{X^{k}\right\}\right)$ Assume that $\mathcal{M}(n)(\beta)$ is positive, recursively generated, and satisfies $Y X=1$. In $\mathcal{S}_{n}(n)$, assume that the first dependence relation occurs at $Y^{k}$, with $1 \leq k<n$. Then $\mathcal{M}(n)$ is flat, and thus admits a unique flat extension $\mathcal{M}(n+1)$.

Proof. Write $Y^{k}=p_{k}(X)+q_{k-1}(Y)$, where $\operatorname{deg} p_{k} \leq k$ and $\operatorname{deg} q_{k-1} \leq k-1$. Since $Y^{k}$ corresponds to a monomial of degree at most $n-1$, and since $Y X=1$ and $\mathcal{M}(n)$ is recursively generated, we must have

$$
Y^{k-1}=X Y^{k}=X p_{k}(X)+X q_{k-1}(Y) .
$$

Since $\mathcal{M}(n)$ is recursively generated and $Y X=1, X q_{k-1}(Y)$ is clearly a linear combination of columns corresponding to monomials of degree at most $k-2$. Let $a_{k}$ be the coefficient of $X^{k}$ in $p_{k}$. If $a_{k}=0$, it follows from (2.3) that $\mathcal{S}_{n}(k-1) \cup\left\{X^{k}\right\}$ is linearly dependent, a contradiction. Thus, we must have $a_{k} \neq 0$, whence (2.3) implies that $X^{k+1}$ is a linear combination of previous columns. Moreover, $Y^{k+1}=$ $Y p_{k}(X)+Y q_{k-1}(Y)$, and $Y p_{k}(X)$ has degree $k-1$ in $X$, so $\mathcal{M}(k+1)$ is flat. It now follows from the Extension Principle [Fia1 and recursiveness that $\mathcal{M}(n)$ is flat, i.e., $\mathcal{M}(n)$ is a flat extension of $\mathcal{M}(k)$.

Proposition 2.7. (Case III: The first dependence relation occurs at $Y^{n}$ ) Assume that $\mathcal{M}(n)(\beta)$ is positive, recursively generated, satisfies $Y X=1$, and $\operatorname{rank} \mathcal{M}(n) \leq$ $\operatorname{card} \mathcal{V}(\beta)$. In $\mathcal{S}_{n}(n)$, assume that $Y^{n}$ is the location of the first dependence relation. Then $\mathcal{M}(n)$ admits a flat extension $\mathcal{M}(n+1)$.

The proof of Proposition 2.7 will require several preliminary results (Lemmas 2.8 2.11 below). Under the hypotheses of Proposition 2.7 write

$$
Y^{n}=a_{n} X^{n}+p_{n-1}(X)+q_{n-1}(Y),
$$

with $\operatorname{deg} p_{n-1}, \operatorname{deg} q_{n-1} \leq n-1$. We claim that $a_{n} \neq 0$. Assume instead that $a_{n}=$ 0 , i.e., $Y^{n}=p_{n-1}(X)+q_{n-1}(Y)$. Then $\mathcal{V}(\beta) \subseteq(y x=1) \bigcap\left(p_{n-1}(x)+q_{n-1}(y)=y^{n}\right)$ $\subseteq(y x=1) \cap\left(p_{n-1}\left(\frac{1}{y}\right)+q_{n-1}(y)=y^{n}\right)$. Since $p_{n-1}\left(\frac{1}{y}\right)+q_{n-1}(y)=y^{n}$ leads to a polynomial equation in $y$ of degree at most $2 n-1$, it follows that card $\mathcal{V}(\beta) \leq 2 n-1$, so $\operatorname{rank} \mathcal{M}(n) \leq 2 n-1$. Then $\mathcal{S}_{n}(n-1) \equiv\left\{1, X, Y, X^{2}, Y^{2}, \ldots, X^{n-1}, Y^{n-1}\right\}$ is a 
basis for $\mathcal{C}_{\mathcal{M}(n)}$, whence $X^{n}$ is a linear combination of the columns in $\mathcal{S}_{n}(n-1)$, a contradiction. Thus, $a_{n} \neq 0$, so in particular

$$
X^{n}=\frac{1}{a_{n}}\left[Y^{n}-p_{n-1}(X)-q_{n-1}(Y)\right] .
$$

To build a flat extension $\mathcal{M}(n+1) \equiv\left(\begin{array}{cc}\mathcal{M}(n) & B(n+1) \\ B(n+1)^{*} & C(n+1)\end{array}\right)$, we define the middle $n$ columns of a prospective block $B \equiv B(n+1)$ by exploiting recursiveness and the relation $Y X=1$, as follows:

$$
Y X^{n}:=X^{n-1} ; Y^{2} X^{n-1}:=Y X^{n-2} ; \ldots, Y^{n} X:=Y^{n-1} .
$$

Also, motivated by (2.5) and, respectively, (2.4), we let

$$
X^{n+1}:=\frac{1}{a_{n}}\left[Y^{n-1}-X p_{n-1}(X)-X q_{n-1}(Y)\right],
$$

and

$$
Y^{n+1}:=a_{n} X^{n-1}+Y p_{n-1}(X)+Y q_{n-1}(Y) .
$$

(The expressions $Y^{n-1}-X p_{n-1}(X)-X q_{n-1}(Y)$ and $a_{n} X^{n-1}+Y p_{n-1}(X)+$ $Y q_{n-1}(Y)$ are shorthand notation for $\left(y^{n-1}-x p_{n-1}(x)-x q_{n-1}(y)\right)(X, Y)$ and $\left(a_{n} x^{n-1}+y p_{n-1}(x)+y q_{n-1}(y)\right)(X, Y)$ in $\mathcal{C}_{\mathcal{M}(n)}$, respectively. Observe that these defining relations are all required if one is to obtain a positive recursively generated moment matrix extension $\mathcal{M}(n+1)$.) Since the columns defined by (2.6) - (2.8) belong to $\mathcal{C}_{\mathcal{M}(n)}$, we have $B=\mathcal{M}(n) W$ for some matrix $W$. Thus, a flat extension $M:=[\mathcal{M}(n) ; B]$ is uniquely determined by defining the $C$-block as $C:=W^{*} \mathcal{M}(n) W$ (cf. Section 1). To complete the proof that $M$ is a moment matrix $\mathcal{M}(n+1)$, it suffices to show that block $B$ is of the form $\left(B_{i, n+1}\right)_{i=0}^{n}$ and that block $C$ is of the form $B_{n+1, n+1}$. To this end, we require some additional notation and several preliminary results.

We next extend the notation $\langle p(X, Y), q(X, Y)\rangle$ to the case when $\operatorname{deg} p=n+1$, $\operatorname{deg} q \leq n$. Indeed, using the definitions of the columns of $B$, for $i, j \geq 0, i+j=$ $n+1$, there exists $p_{i j} \in \mathbb{R}_{n}[x, y]$ with $Y^{i} X^{j}=p_{i j}(X, Y)$, and we define

$$
\left\langle Y^{i} X^{j}, q(X, Y)\right\rangle:=\left\langle p_{i j}(X, Y), q(X, Y)\right\rangle .
$$

Now if $p(x, y) \equiv \sum_{0 \leq k+\ell \leq n+1} a_{k \ell} x^{\ell} y^{k}$, we define

$$
\langle p(X, Y), q(X, Y)\rangle:=\sum_{0 \leq k+\ell \leq n+1} a_{k \ell}\left\langle Y^{k} X^{\ell}, q(X, Y)\right\rangle .
$$

It is easy to check that Lemma 2.3 (iii) holds with $\operatorname{deg} r=n+1$.

Lemma 2.8. Under the hypotheses of Proposition 2.7. assume $i, j \geq 0$, with $i+j=$ $n+1$, and $r, s \geq 1$, with $r+s \leq n$. Then

$$
\left\langle Y^{i} X^{j}, Y^{r} X^{s}\right\rangle=\left\langle Y^{i} X^{j}, Y^{r-1} X^{s-1}\right\rangle .
$$


Proof. Fix $i$ and $j$ with $i+j=n+1$. We know from (2.6) - (2.8) that there exists a polynomial $p \in \mathbb{R}_{n}[x, y]$ such that $Y^{i} X^{j}=p(X, Y) \equiv \sum_{k+\ell \leq n} a_{k, \ell} Y^{k} X^{\ell}$. Then

$$
\begin{aligned}
\left\langle Y^{i} X^{j}, Y^{r} X^{s}\right\rangle & =\sum_{k+\ell \leq n} a_{k, \ell}\left\langle Y^{k} X^{\ell}, Y^{r} X^{s}\right\rangle \\
& =\sum_{k+\ell \leq n} a_{k, \ell}\left\langle Y^{r} X^{s}, Y^{k} X^{\ell}\right\rangle \quad \text { (because } \mathcal{M}(n) \text { is self-adjoint) } \\
& =\sum_{k+\ell \leq n} a_{k, \ell}\left\langle Y^{r-1} X^{s-1}, Y^{k} X^{\ell}\right\rangle \quad \text { (using } Y X=1 \text { and recursiveness) } \\
& =\sum_{k+\ell \leq n} a_{k, \ell}\left\langle Y^{k} X^{\ell}, Y^{r-1} X^{s-1}\right\rangle
\end{aligned}
$$

(using again the self-adjointness of $\mathcal{M}(n)$ )

$$
=\left\langle Y^{i} X^{j}, Y^{r-1} X^{s-1}\right\rangle \text {, }
$$

as desired.

The next result provides a reduction for the proof that $B(n+1)$ has the Hankel property.

Lemma 2.9. Under the hypotheses of Proposition 2.7 assume $i+j=n+1$, with $j \geq 1, i \geq 0$, and assume that the Hankel property

$$
\left\langle Y^{i} X^{j}, Y^{r} X^{s}\right\rangle=\left\langle Y^{i+1} X^{j-1}, Y^{r-1} X^{s+1}\right\rangle
$$

holds with $1 \leq r \leq n$ and $s=0$. Then 2.10) holds for all $r$ and $s$ such that $1 \leq r+s \leq n, r \geq 1, s \geq 0$.

Proof. Fix $i$ and $j$ with $i+j=n+1$. We use induction on $t:=r+s$, where $1 \leq r+s \leq n, r \geq 1, s \geq 0$. For $t=1$ the result follows from the hypothesis, since $r=1, s=0$. Assume now that $t=2$. By hypothesis, we may assume $r=s=1$, so we consider the equation

$$
\left\langle Y^{i} X^{j}, Y X\right\rangle=\left\langle Y^{i+1} X^{j-1}, X^{2}\right\rangle,
$$

with $j \geq 1, i \geq 0, i+j=n+1$. Since $Y X=1$, the left-hand side of (2.11) equals $\left\langle Y^{i} X^{j}, 1\right\rangle$ by Lemma 2.8 For $j \geq 2$ and $i \geq 1$, the right-hand side of (2.11) equals $\left\langle Y^{i} X^{j-2}, X^{2}\right\rangle$ (by (2.6)), which in turn equals $\left\langle Y^{i} X X^{j-2}, X\right\rangle=\left\langle Y^{i-1} X^{j-2}, X\right\rangle=$ $\left\langle Y^{i-1} X^{j-1}, 1\right\rangle=\left\langle Y^{i} X^{j}, 1\right\rangle$ (by (2.6) for the last step). When $j \geq 2$ and $i=0$ (which then implies $j=n+1$ ), we have

$$
\begin{aligned}
\left\langle X^{n+1}, Y X\right\rangle & =\left\langle\frac{1}{a_{n}}\left[Y^{n-1}-X p_{n-1}(X)-X q_{n-1}(Y)\right], Y X\right\rangle \text { (by 2.7) } \\
& =\frac{1}{a_{n}}\left[\left\langle Y^{n}, X\right\rangle-\left\langle p_{n-1}(X), X Y X\right\rangle-\left\langle q_{n-1}(Y), X Y X\right\rangle\right]
\end{aligned}
$$

(by Lemma 2.3(ii) for the first term and Lemma 2.4 for the last two terms)

$$
\begin{aligned}
& =\left\langle\frac{1}{a_{n}}\left[Y^{n}-p_{n-1}(X)-q_{n-1}(Y)\right], X\right\rangle \text { (by Lemma 2.3(iii)) } \\
& \left.=\left\langle X^{n}, X\right\rangle \text { (by (2.5) }\right) \\
& =\left\langle X^{n-1}, X^{2}\right\rangle \\
& \left.=\left\langle Y X^{n}, X^{2}\right\rangle \text { (by 2.6) }\right) .
\end{aligned}
$$


When $j=1$ (so that $i=n$ ), the right-hand side of (2.11) is

$$
\begin{aligned}
\left\langle Y^{n+1}, X^{2}\right\rangle & \left.=\left\langle a_{n} X^{n-1}+Y p_{n-1}(X)+Y q_{n-1}(Y), X^{2}\right\rangle \quad \text { (by 2.8) }\right) \\
& =\left\langle a_{n} X^{n}+p_{n-1}(X)+q_{n-1}(Y), X\right\rangle \\
& \text { (using Lemma 2.3(ii) and Lemma 2.4] as above) } \\
& \left.=\left\langle Y^{n}, X\right\rangle \quad \text { (by (2.4) }\right) \\
& =\left\langle Y^{n-1}, 1\right\rangle \quad \text { (again using Lemma 2.4). }
\end{aligned}
$$

On the other hand, the left-hand side of (2.11) is

$$
\begin{aligned}
\left\langle Y^{n} X, Y X\right\rangle & =\left\langle Y^{n-1}, Y X\right\rangle(\text { by (2.6) }) \\
& =\left\langle Y^{n-1}, 1\right\rangle \quad \text { (by Lemma 2.3)(iii). }
\end{aligned}
$$

This completes the case when $t=2$.

Assume now that (2.10) is true for $t \leq u$, with $u \geq 2$, and consider the case $t=u+1$. Thus $r+s=u+1(\leq n)$, and we may assume $r, s \geq 1$. When $r \geq 2$,

$$
\begin{aligned}
\left\langle Y^{i} X^{j}, Y^{r} X^{s}\right\rangle & =\left\langle Y^{i} X^{j}, Y^{r-1} X^{s-1}\right\rangle \quad \text { (by Lemma 2.8) } \\
& =\left\langle Y^{i+1} X^{j-1}, Y^{r-2} X^{s}\right\rangle \quad \text { (by the inductive step) } \\
& =\left\langle Y^{i+1} X^{j-1}, Y^{r-1} X^{s+1}\right\rangle \quad \text { (by Lemma 2.8) }
\end{aligned}
$$

as desired. When $r=1$ and $j \geq 2$, we have $s \leq n-r=n-1$, and we consider three subcases.

Subcase 1. For $j=2, i=n-1$,

$$
\begin{aligned}
\left\langle Y^{n-1} X^{2}, Y X^{s}\right\rangle & =\left\langle Y^{n-2} X, Y X^{s}\right\rangle \quad(\text { by (2.6) }) \\
& =\left\langle Y^{n-2} X, X^{s-1}\right\rangle \quad(\text { by Lemma 2.3(iii) }) \\
& =\left\langle Y^{n-2}, X^{s}\right\rangle \\
& =\left\langle Y^{n-1}, X^{s+1}\right\rangle \quad(\text { by Lemma 2.4 since } s \leq n-1) \\
& =\left\langle Y^{n} X, X^{s+1}\right\rangle \quad(\text { by (2.6) }) .
\end{aligned}
$$

Subcase 2. For $j \geq 3, i \geq 1$,

$$
\begin{aligned}
\left\langle Y^{i} X^{j}, Y X^{s}\right\rangle & \left.=\left\langle Y^{i-1} X^{j-1}, Y X^{s}\right\rangle \text { (by (2.6) }\right) \\
& =\left\langle Y^{i-1} X^{j-1}, X^{s-1}\right\rangle \text { (by Lemma 2.3(iii)) } \\
& \left.=\left\langle Y^{i-1} X^{j-3}, X^{s+1}\right\rangle=\left\langle Y^{i} X^{j-2}, X^{s+1}\right\rangle \text { (since } Y X=1 \text { in } \mathcal{M}(n)\right) \\
& \left.=\left\langle Y^{i+1} X^{j-1}, X^{s+1}\right\rangle \text { (by (2.6) }\right) .
\end{aligned}
$$

Subcase 3. For $j=n+1, i=0$,

$$
\begin{aligned}
\left\langle X^{n+1}, Y X^{s}\right\rangle & =\left\langle\frac{1}{a_{n}}\left[Y^{n-1}-X p_{n-1}(X)-X q_{n-1}(Y)\right], Y X^{s}\right\rangle \\
& =\left\langle\frac{1}{a_{n}}\left[Y^{n}-p_{n-1}(X)-q_{n-1}(Y)\right], X^{s}\right\rangle \text { (by Lemma 2.4) } \\
& =\left\langle X^{n}, X^{s}\right\rangle=\left\langle X^{n-1}, X^{s+1}\right\rangle \\
& =\left\langle Y X^{n}, X^{s+1}\right\rangle(\text { by (2.6) }) .
\end{aligned}
$$


Finally, when $r=1$ and $j=1$, we have $i=n$ and $s \leq n-1$, so

$$
\begin{aligned}
\left\langle Y^{n} X, Y X^{s}\right\rangle & \left.=\left\langle Y^{n-1}, Y X^{s}\right\rangle \quad \text { by (2.6) }\right) \\
& \left.=\left\langle Y^{n}, X^{s}\right\rangle=\left\langle a_{n} X^{n}+p_{n-1}(X)+q_{n-1}(Y), X^{s}\right\rangle \quad \text { by (2.4) }\right) \\
& =\left\langle a_{n} X^{n-1}+Y p_{n-1}(X)+Y q_{n-1}(Y), X^{s+1}\right\rangle
\end{aligned}
$$

(by Lemma 2.3(ii) and Lemma 2.4 as above)

$$
=\left\langle Y^{n+1}, X^{s+1}\right\rangle \text {. }
$$

Recall from (2.6) that columns $Y X^{n}, \ldots, Y^{n} X$ are taken as a block from consecutive columns of degree $n-1$ in $\mathcal{M}(n)$, so these columns satisfy the Hankel property. Thus, in view of Lemma 2.9] the next two results complete the proof that $B(n+1)$ has the Hankel property.

Lemma 2.10. For $k=1, \ldots, n$,

$$
\left\langle X^{n+1}, Y^{k}\right\rangle=\left\langle Y X^{n}, Y^{k-1} X\right\rangle \text {. }
$$

Proof. We have

$$
\begin{aligned}
\left\langle X^{n+1}, Y^{k}\right\rangle & =\left\langle\frac{1}{a_{n}}\left[Y^{n-1}-X p_{n-1}(X)-X q_{n-1}(Y)\right], Y^{k}\right\rangle \quad \text { (by (2.7)) } \\
& =\left\langle\frac{1}{a_{n}}\left[Y^{n}-p_{n-1}(X)-q_{n-1}(Y)\right], Y^{k-1}\right\rangle
\end{aligned}
$$

(by Lemma 2.3 (ii) and Lemma 2.4 as above)

$$
\begin{aligned}
& =\left\langle X^{n}, Y^{k-1}\right\rangle=\left\langle X^{n-1}, Y^{k-1} X\right\rangle \\
& \left.=\left\langle Y X^{n}, Y^{k-1} X\right\rangle \quad \text { by (2.6) }\right) .
\end{aligned}
$$

Lemma 2.11. For $k=1, \ldots, n$,

$$
\left\langle Y^{n} X, Y^{k}\right\rangle=\left\langle Y^{n+1}, Y^{k-1} X\right\rangle \text {. }
$$

Proof. We have

$$
\begin{aligned}
\left\langle Y^{n} X, Y^{k}\right\rangle & =\left\langle Y^{n-1}, Y^{k}\right\rangle \quad \text { by 2.6) } \\
& \left.=\left\langle Y^{n}, Y^{k-1}\right\rangle=\left\langle a_{n} X^{n}+p_{n-1}(X)+q_{n-1}(Y), Y^{k-1}\right\rangle \text { (by (2.4) }\right) \\
& =\left\langle a_{n} X^{n-1}+Y p_{n-1}(X)+Y q_{n-1}(Y), Y^{k-1} X\right\rangle
\end{aligned}
$$

(by Lemma 2.4 for the last two terms)

$$
\left.=\left\langle Y^{n+1}, Y^{k-1} X\right\rangle \text { (by (2.8) }\right)
$$

The proof that block $B$ is of the form $\left\{B_{i, n+1}\right\}_{i=0}^{n}$ is now complete. To finish the proof of Proposition [2.7 it now suffices to show that $C:=W^{*} \mathcal{M}(n) W$ is Hankel. To do this, observe that in the $C$ block of $\mathcal{M}:=[\mathcal{M}(n) ; B]=\left(\begin{array}{cc}\mathcal{M}(n) & B \\ B^{*} & C\end{array}\right)$, we need to compute inner products of the form $\left\langle Y^{i} X^{j}, Y^{k} X^{\ell}\right\rangle \quad(i+j=k+\ell=$ $n+1)$. For this, we require an auxiliary lemma. For $i+j=k+\ell=n+1$, by $\left\langle Y^{i} X^{j}, Y^{k} X^{\ell}\right\rangle$ we mean, as usual, the entry in row $Y^{k} X^{\ell}$ of column $Y^{i} X^{j}$; by self-adjointness of $\mathcal{M}(n+1)$, we have $\left\langle Y^{i} X^{j}, Y^{k} X^{\ell}\right\rangle=\left\langle Y^{k} X^{\ell}, Y^{i} X^{j}\right\rangle$. Now 
suppose $Y^{i} X^{j}=p(X, Y)$ and $Y^{k} X^{\ell}=q(X, Y)$, where $p(x, y) \equiv \sum_{0 \leq r+s \leq n} a_{r s} y^{r} x^{s}$ and $q(x, y) \equiv \sum_{0 \leq t+u \leq n} b_{t u} y^{t} x^{u}$; we define $\langle p(X, Y), q(X, Y)\rangle:=$ $\sum_{0 \leq r+s, t+u \leq n} a_{r s} b_{t u}\left\langle Y^{r} X^{s}, Y^{t} X^{u}\right\rangle$.

Lemma 2.12. For $i+j, k+\ell=n+1,\left\langle Y^{i} X^{j}, Y^{k} X^{\ell}\right\rangle=\langle p(X, Y), q(X, Y)\rangle$.

Proof.

$$
\begin{aligned}
\langle p(X, Y), q(X, Y)\rangle & \equiv \sum_{0 \leq r+s, t+u \leq n} a_{r s} b_{t u}\left\langle Y^{r} X^{s}, Y^{t} X^{u}\right\rangle \\
& =\sum_{0 \leq t+u \leq n} b_{t u}\left\langle\sum_{0 \leq r+s \leq n} a_{r s} Y^{r} X^{s}, Y^{t} X^{u}\right\rangle \\
& =\sum_{0 \leq t+u \leq n} b_{t u}\left\langle p(X, Y), Y^{t} X^{u}\right\rangle=\sum_{0 \leq t+u \leq n} b_{t u}\left\langle Y^{i} X^{j}, Y^{t} X^{u}\right\rangle \\
& =\sum_{0 \leq t+u \leq n} b_{t u}\left\langle Y^{t} X^{u}, Y^{i} X^{j}\right\rangle \quad\left(\text { since } \mathcal{M}=\mathcal{M}^{t}\right) \\
& =\left\langle\sum_{0 \leq t+u \leq n} b_{t u} Y^{t} X^{u}, Y^{i} X^{j}\right\rangle=\left\langle q(X, Y), Y^{i} X^{j}\right\rangle \\
& \left.=\left\langle Y^{k} X^{\ell}, Y^{i} X^{j}\right\rangle=\left\langle Y^{i} X^{j}, Y^{k} X^{\ell}\right\rangle \quad \text { (since } \mathcal{M}=\mathcal{M}^{t}\right) .
\end{aligned}
$$

Proof of Proposition 2.7. Note that since $M$ is a flat extension, dependence relations in the columns of $(\mathcal{M}(n) \quad B)$ extend to column relations in $\left(\begin{array}{ll}B^{*} & C\end{array}\right)$. In particular, the middle $n$ columns of $C$ coincide with the columns of degree $n-1$ of $B^{*}$; since $B$ has the Hankel property, so does $B^{*}$, and thus the middle $n$ columns of $C$ have the Hankel property. To verify that $C$ is Hankel it now suffices to focus on the first two and the last two columns of $C$, namely $X^{n+1}$ and $Y X^{n}$, and $Y^{n} X$ and $Y^{n+1}$. Since $C$ is self-adjoint, and the middle $n$ columns have the Hankel property, to check that $C$ is Hankel it only remains to show that $C_{n+2,1}=C_{n+1,2}$, i.e., $\left\langle X^{n+1}, Y^{n+1}\right\rangle=\left\langle Y X^{n}, Y^{n} X\right\rangle$. Now, by (2.7), 2.8) and Lemma 2.12 we have

$$
\begin{aligned}
& \left\langle X^{n+1}, Y^{n+1}\right\rangle \\
& =\left\langle\frac{1}{a_{n}}\left\{Y^{n-1}-X\left[p_{n-1}(X)+q_{n-1}(Y)\right]\right\}, a_{n} X^{n-1}+Y\left[p_{n-1}(X)+q_{n-1}(Y)\right]\right\rangle \\
& =\left\langle Y^{n-1}, X^{n-1}\right\rangle+\frac{1}{a_{n}}\left\langle Y^{n-1}, Y\left[p_{n-1}(X)+q_{n-1}(Y)\right]\right\rangle \\
& -\left\langle X\left[p_{n-1}(X)+q_{n-1}(Y)\right], X^{n-1}\right\rangle \\
& -\frac{1}{a_{n}}\left\langle X\left[p_{n-1}(X)+q_{n-1}(Y)\right], Y\left[p_{n-1}(X)+q_{n-1}(Y)\right]\right\rangle \\
& =\left\langle Y^{n}, X^{n}\right\rangle+\frac{1}{a_{n}}\left\langle Y^{n}, p_{n-1}(X)+q_{n-1}(Y)\right\rangle-\left\langle p_{n-1}(X)+q_{n-1}(Y), X^{n}\right\rangle \\
& \left.\left.-\frac{1}{a_{n}}\left\langle p_{n-1}(X)+q_{n-1}(Y)\right], p_{n-1}(X)+q_{n-1}(Y)\right]\right\rangle
\end{aligned}
$$

(by Lemma 2.4 for the first and fourth terms, and Lemma 2.3(ii) for the second and third terms) 


$$
\begin{aligned}
& =\left\langle\frac{1}{a_{n}}\left\{Y^{n}-\left[p_{n-1}(X)+q_{n-1}(Y)\right]\right\}, a_{n} X^{n}+p_{n-1}(X)+q_{n-1}(Y)\right\rangle \\
& =\left\langle X^{n}, Y^{n}\right\rangle \text { (by 2.5), 2.4), and Lemma2.3(i),(iii)) } \\
& =\left\langle X^{n-1}, Y^{n-1}\right\rangle \quad \text { (by Lemma 2.4) } \\
& =\left\langle Y X^{n}, Y^{n} X\right\rangle \quad \text { (by (2.6) and Lemma 2.12). }
\end{aligned}
$$

This concludes the proof of Proposition 2.7

Remark 2.13. It is important for the sequel to note that in the proof of Proposition 2.7 the variety condition $\operatorname{rank} \mathcal{M}(n) \leq \operatorname{card} \mathcal{V}(\mathcal{M}(n))$ was used only to show that $a_{n} \neq 0$ in (2.4). Thus, if $\mathcal{M}(n)$ is positive, recursively generated, satisfies $Y X=1$ in $\mathcal{C}_{\mathcal{M}(n)}$, and if the first dependence relation in $\mathcal{S}_{n}(n)$ is of the form (2.4) with $a_{n} \neq 0$, then we may conclude that $\mathcal{M}(n)$ has a flat extension $\mathcal{M}(n+1)$.

Proposition 2.14. (Case IV: $\operatorname{rank} \mathcal{M}(n)=2 n+1$ ) Assume that $\mathcal{M}(n)$ is positive, recursively generated, and satisfies $Y X=1$. Assume also that $\mathcal{S}_{n}(n)$ is a basis for $\mathcal{C}_{\mathcal{M}(n)}$. Then $\mathcal{M}(n)$ admits a flat extension $\mathcal{M}(n+1)$, or $\mathcal{M}(n)$ admits a positive, recursively generated extension $\mathcal{M}(n+1)$, with $\operatorname{rank} \mathcal{M}(n+1)=2 n+2$, and $\mathcal{M}(n+1)$ admits a flat extension $\mathcal{M}(n+2)$.

Proof. Since $Y X=1$, and to guarantee that $\mathcal{M}(n+1)$ is recursively generated, we define the middle $n$ columns of a proposed $B$ block for $\mathcal{M}(n+1)$ as $\left[Y X^{n}\right]_{m(n)}:=$ $X^{n-1} \in \mathcal{C}_{\mathcal{M}(n)},\left[Y^{2} X^{n-1}\right]_{m(n)}:=Y X^{n-2} \in \mathcal{C}_{\mathcal{M}(n)}, \ldots,\left[Y^{n} X\right]_{m(n)}:=Y^{n-1} \in$ $\mathcal{C}_{\mathcal{M}(n)}$. Moreover, if we wish to make $B_{n, n+1}$ Hankel, it is clear that all but the entry $\left\langle X^{n+1}, X^{n}\right\rangle$ in the column $\left[X^{n+1}\right]_{m(n)}$ must be given in terms of entries in $\mathcal{M}(n)$, and that all but the entry $\left\langle Y^{n+1}, Y^{n}\right\rangle$ in $\left[Y^{n+1}\right]_{m(n)}$ must be given in terms of entries in $\mathcal{M}(n)$. To handle the remaining entries we introduce two parameters $p$ and $q$; concretely, for $i+j=0, \ldots, n$,

$$
\begin{gathered}
\left\langle X^{n+1}, Y^{i} X^{j}\right\rangle:=\left\{\begin{array}{cc}
\left\langle Y X^{n}, Y^{i-1} X^{j+1}\right\rangle & (1 \leq i \leq n) \\
\beta_{0, n+j+1} & (i=0,0 \leq j \leq n-1) \\
p & (i=0, j=n)
\end{array}\right. \\
\left\langle Y^{n+1}, Y^{i} X^{j}\right\rangle:=\left\{\begin{array}{cc}
\left\langle Y^{n} X, Y^{i+1} X^{j-1}\right\rangle & (1 \leq j \leq n) \\
\beta_{n+1+i, 0} & (0 \leq i \leq n-1, j=0) \\
q & (i=n, j=0)
\end{array} .\right.
\end{gathered}
$$

A positive extension $\mathcal{M}(n+1)$ entails $\operatorname{Ran} B \subseteq \operatorname{Ran} \mathcal{M}(n)$, so in particular we must show that $\left[X^{n+1}\right]_{m(n)},\left[Y^{n+1}\right]_{m(n)} \in \operatorname{Ran} \mathcal{M}(n)$. To this end, note that since $N:=[\mathcal{M}(n)]_{\mathcal{S}_{n}(n)}>0$, there exist vectors $\mathbf{f}, \mathbf{g} \in \mathbb{R}^{2 n+1}$ such that $N \mathbf{f}=$ $\left[X^{n+1}\right]_{\mathcal{S}_{n}(n)}$ and $N \mathbf{g}=\left[Y^{n+1}\right]_{\mathcal{S}_{n}(n)}$. Let $\mathbf{F}, \mathbf{G} \in \mathbb{R}^{m(n)}$ be given by

$$
\left\langle\mathbf{F}, Y^{i} X^{j}\right\rangle:=\left\{\begin{array}{cl}
\left\langle\mathbf{f}, Y^{i} X^{j}\right\rangle & \text { if } Y^{i} X^{j} \in \mathcal{S}_{n}(n) \\
0 & \text { otherwise }
\end{array}\right.
$$

and

$$
\left\langle\mathbf{G}, Y^{i} X^{j}\right\rangle:=\left\{\begin{array}{cl}
\left\langle\mathbf{g}, Y^{i} X^{j}\right\rangle & \text { if } Y^{i} X^{j} \in \mathcal{S}_{n}(n) \\
0 & \text { otherwise }
\end{array} .\right.
$$

We observe, for future reference, that since $\mathbf{f}=N^{-1}\left[X^{n+1}\right]_{\mathcal{S}_{n}(n)}, \mathbf{f}$ is linear in $p$ (and independent of $q$ ), and so also is $\mathbf{F}$; similarly, $\mathbf{g}$ and $\mathbf{G}$ are linear in $q$ and independent of $p$. 
Claim. $\mathcal{M}(n) \mathbf{F}=\left[X^{n+1}\right]_{m(n)} ;$ equivalently,

$$
\left\langle\mathcal{M}(n) \mathbf{F}, Y^{i} X^{j}\right\rangle=\left\langle\left[X^{n+1}\right]_{m(n)}, Y^{i} X^{j}\right\rangle
$$

for each $(i, j) \in I_{u}:=\{(i, j): i+j \leq n$ and $((i=u \leq j \leq n)$ or $(j=u \leq i \leq n))\}$, $u=0,1, \ldots,\left[\frac{n}{2}\right]$. Our proof of Claim 1 is by induction on $u$. For $u=0$, we consider $Z \equiv Y^{i}$ or $Z \equiv X^{j}$ in $\mathcal{S}_{n}(n)$, so

$$
\begin{aligned}
\langle\mathcal{M}(n) \mathbf{F}, Z\rangle & =\sum_{Y^{k} X^{\ell} \in \mathcal{C}_{\mathcal{M}(n)}}\left\langle Y^{k} X^{\ell}, Z\right\rangle\left\langle\mathbf{F}, Y^{k} X^{\ell}\right\rangle \\
& =\sum_{Y^{k} X^{\ell} \in \mathcal{S}_{n}(n)}\left\langle Y^{k} X^{\ell}, Z\right\rangle\left\langle\mathbf{f}, Y^{k} X^{\ell}\right\rangle+\sum_{Y^{k} X^{\ell} \notin \mathcal{S}_{n}(n)}\left\langle Y^{k} X^{\ell}, Z\right\rangle \cdot 0 \\
& =\langle N \mathbf{f}, Z\rangle=\left\langle\left[X^{n+1}\right]_{m(n)}, Z\right\rangle,
\end{aligned}
$$

as desired. We must now deal with rows of the form $Y^{i} X^{j}(i, j \geq 1)$; that is, we must prove that $\left\langle\mathcal{M}(n) \mathbf{F}, Y^{i} X^{j}\right\rangle=\left\langle\left[X^{n+1}\right]_{m(n)}, Y^{i} X^{j}\right\rangle$ for $i, j \geq 1$ and $i+j \leq n$. Assume that the Claim is true for $u=k\left(0 \leq k \leq\left[\frac{n}{2}\right]-1\right)$, and consider $(i, j) \in I_{k+1}$. We have

$$
\begin{aligned}
\left\langle\left[X^{n+1}\right]_{m(n)}, Y^{i} X^{j}\right\rangle & \equiv\left\langle X^{n+1}, Y^{i} X^{j}\right\rangle=\left\langle Y X^{n}, Y^{i-1} X^{j+1}\right\rangle \quad \text { by 2.13) } \\
& =\left\langle X^{n-1}, Y^{i-1} X^{j+1}\right\rangle .
\end{aligned}
$$

On the other hand,

$$
\begin{aligned}
\left\langle\mathcal{M}(n) \mathbf{F}, Y^{i} X^{j}\right\rangle & =\left\langle\mathcal{M}(n) \mathbf{F}, Y^{i-1} X^{j-1}\right\rangle \quad \text { (by Lemma[2.3(iii)) } \\
& =\left\langle\left[X^{n+1}\right]_{m(n)}, Y^{i-1} X^{j-1}\right\rangle \text { (by the inductive step) } \\
& =\left\langle X^{n+1}, Y^{i-1} X^{j-1}\right\rangle .
\end{aligned}
$$

It thus suffices to prove that $\left\langle X^{n+1}, Y^{i-1} X^{j-1}\right\rangle=\left\langle X^{n-1}, Y^{i-1} X^{j+1}\right\rangle$. For $i=1$,

$$
\begin{aligned}
\left\langle X^{n+1}, X^{j-1}\right\rangle & =\beta_{0, n+j}(\text { by }(2.131) \\
& =\left\langle X^{n-1}, X^{j+1}\right\rangle,
\end{aligned}
$$

and for $i>1$,

$$
\begin{aligned}
\left\langle X^{n+1}, Y^{i-1} X^{j-1}\right\rangle & =\left\langle Y X^{n}, Y^{i-2} X^{j}\right\rangle \text { (by 2.13) } \\
& =\left\langle X^{n-1}, Y^{i-2} X^{j}\right\rangle=\left\langle X^{n-1}, Y^{i-1} X^{j+1}\right\rangle \quad \text { (by Lemma 2.3(iii). }
\end{aligned}
$$

This completes the proof of the Claim. An entirely similar argument, using $\mathbf{g}$ instead of $\mathbf{f}$ and (2.14) instead of (2.13), shows that $\mathcal{M}(n) \mathbf{G}=\left[Y^{n+1}\right]_{m(n)}$. Moreover, by definition, $\left[Y^{i} X^{j}\right]_{m(n)}=Y^{i-1} X^{j-1} \in \mathcal{C}_{\mathcal{M}(n)}(i+j=n+1 ; i, j \geq 1)$, so we now have $\operatorname{Ran} B \subseteq \operatorname{Ran} \mathcal{M}(n)$; in particular, there exists $W$ such that $\mathcal{M}(n) W=B$.

We note the following for future reference. From Lemma 2.2 and the fact that $\mathcal{M}(n)=\mathcal{M}(n)^{t}$, each row of $\mathcal{M}(n)$ coincides with a row indexed by an element of $\mathcal{S}_{n}(n)$. Since $B=\mathcal{M}(n) W$, it now follows that each row of $(\mathcal{M}(n) B)$ coincides with a row of $(\mathcal{M}(n) B)$ indexed by an element of $\mathcal{S}_{n}(n)$. 
We now form the flat extension $\mathcal{M}:=[\mathcal{M}(n) ; B] \equiv\left(\begin{array}{cc}\mathcal{M}(n) & B \\ B^{t} & C\end{array}\right)$, where $C:=W^{t} \mathcal{M}(n) W$. Exactly as in the proof of Proposition 2.7 $C$ is of the form

$$
C \equiv\left(\begin{array}{ccccc}
\tau & \beta_{0,2 n} & \cdots & \beta_{02} & \eta \\
\beta_{0,2 n} & \beta_{0,2 n-2} & \cdots & \beta_{00} & \beta_{20} \\
\vdots & \vdots & \ddots & \vdots & \vdots \\
\beta_{02} & \beta_{00} & \cdots & \beta_{2 n-2,0} & \beta_{2 n, 0} \\
\eta & \beta_{20} & \cdots & \beta_{2 n, 0} & \rho
\end{array}\right)
$$

where $C_{11} \equiv \tau:=\left[X^{n+1}\right]_{\mathcal{S}_{n}(n)}^{t} N^{-1}\left[X^{n+1}\right]_{\mathcal{S}_{n}(n)}$ and $C_{1, n+2}=C_{n+2,1} \equiv \eta:=$ $\left[X^{n+1}\right]_{\mathcal{S}_{n}(n)}^{t} N^{-1}\left[Y^{n+1}\right]_{\mathcal{S}_{n}(n)}$. Thus, if $\eta=\beta_{00}$, then $\mathcal{M}$ is a flat moment matrix extension of the form $\mathcal{M}(n+1)$, and we are done.

Assume now that $\eta \neq \beta_{00}$. Let $u>\tau$ be arbitrary, and consider the moment matrix $\mathcal{M}^{\prime} \equiv \mathcal{M}(n+1)^{\prime}$ obtained from $\mathcal{M}$ by replacing $\tau$ by $u$ and $\eta$ by $\beta_{00}$. We partition $\mathcal{M}^{\prime}$ as $\mathcal{M}^{\prime} \equiv\left(\begin{array}{cc}\tilde{\mathcal{M}} & \tilde{B} \\ \tilde{B}^{t} & \tilde{C}\end{array}\right)$, where $\tilde{\mathcal{M}}$ is the compression of $\mathcal{M}^{\prime}$ to rows and columns indexed by $\tilde{\mathcal{B}}:=\left\{1, X, Y, X^{2}, Y X, Y^{2}, \ldots, X^{n}, Y X^{n-1}, \ldots\right.$, $\left.Y^{n-1} X, Y^{n}, X^{n+1}\right\} \subseteq \mathcal{C}_{\mathcal{M}^{\prime}}$ (i.e., $\tilde{\mathcal{M}}$ is the extension of $\mathcal{M}(n)$ by row $X^{n+1}$ and column $X^{n+1}$ of $\left.\mathcal{M}^{\prime}\right)$.

We claim that Ran $\tilde{B} \subseteq \operatorname{Ran} \tilde{\mathcal{M}}$. By the flat construction of $\mathcal{M}$, the "middle" columns of $\left(\begin{array}{c}B \\ C\end{array}\right)$ are borrowed from columns of $\left(\begin{array}{c}\mathcal{M}(n) \\ B^{t}\end{array}\right)$ of degree $n-1$, so, in particular, the columns of $\tilde{B}$ (except the rightmost column) are borrowed from columns in $\tilde{\mathcal{M}}$. To prove the claim, it thus suffices to show that $\left[Y^{n+1}\right]_{\tilde{\mathcal{B}}} \in \operatorname{Ran} \tilde{\mathcal{M}}$. Since $u>\tau, \tilde{\mathcal{S}}:=\left\{1, X, Y, X^{2}, Y^{2}, \ldots, X^{n}, Y^{n}, X^{n+1}\right\}$ is a basis for $\mathcal{C}_{\tilde{\mathcal{M}}}$, and $[\tilde{\mathcal{M}}]_{\tilde{\mathcal{S}}}$ is positive and invertible. Thus there exist unique scalars $a_{1}, a_{2}, \ldots, a_{2 n+2}$ such that, in $\mathcal{C}_{(\tilde{\mathcal{M}} \tilde{B})}$, we have

$$
\left[Y^{n+1}\right]_{\tilde{\mathcal{S}}}=a_{1}[1]_{\tilde{\mathcal{S}}}+a_{2}[X]_{\tilde{\mathcal{S}}}+\ldots+a_{2 n+1}\left[Y^{n}\right]_{\tilde{\mathcal{S}}}+a_{2 n+2}\left[X^{n+1}\right]_{\tilde{\mathcal{S}}}
$$

From the first part of the proof (concerning block $B$ ), we know that each row of $(\tilde{\mathcal{M}} \tilde{B})$ coincides with a row indexed by an element of $\tilde{\mathcal{S}}$, so it now follows that, in $\mathcal{C}_{(\tilde{\mathcal{M}} \tilde{B})}$,

$$
\left[Y^{n+1}\right]_{\tilde{\mathcal{B}}}=a_{1}[1]_{\tilde{\mathcal{B}}}+a_{2}[X]_{\tilde{\mathcal{B}}}+\ldots+a_{2 n+1}\left[Y^{n}\right]_{\tilde{\mathcal{B}}}+a_{2 n+2}\left[X^{n+1}\right]_{\tilde{\mathcal{B}}}
$$

whence the claim is proved.

Since $\tilde{\mathcal{M}} \geq 0$ and $\operatorname{Ran} \tilde{B} \subseteq \operatorname{Ran} \tilde{\mathcal{M}}$, we may construct the (positive) flat extension $\mathcal{M}^{b}:=[\tilde{\mathcal{M}} ; \tilde{B}] \equiv\left(\begin{array}{cc}\tilde{\mathcal{M}} & \tilde{B} \\ \tilde{B}^{t} & D\end{array}\right)$, which we may re-partition as the moment matrix $\mathcal{M}(n+1)=\left(\begin{array}{cc}\mathcal{M}(n) & B \\ B^{t} & C^{b}\end{array}\right)$, where $C^{b}$ is obtained from $C$ by replacing $\tau$ by $u, \eta$ by $\beta_{00}$, and $\rho$ by some $\rho^{b}$ (determined by extending (2.15) to the full columns of $\left.\mathcal{M}^{b}\right)$.

Now $\mathcal{M}(n+1)$ is positive, recursively generated, satisfies $Y X=1$, and (by flatness of $\left.\mathcal{M}^{b}\right), \operatorname{rank} \mathcal{M}(n+1)=\operatorname{rank} \tilde{\mathcal{M}}=1+\operatorname{rank} \mathcal{M}(n)$. In $\mathcal{S}_{n+1}(n+1)$, the first dependence relation is of the form $Y^{n+1}=a_{1} 1+a_{2} X+\ldots+a_{2 n+1} Y^{n}+a_{2 n+2} X^{n+1}$, and we assert that $a_{2 n+2} \neq 0$. Indeed, if $a_{2 n+2}=0$, then $\left[Y^{n+1}\right]_{\mathcal{S}_{n}(n)}=a_{1}[1]_{\mathcal{S}_{n}(n)}+$ 
$a_{2}[X]_{\mathcal{S}_{n}(n)}+\ldots+a_{2 n+1}\left[Y^{n}\right]_{\mathcal{S}_{n}(n)}$, whence $\left(a_{1}, \ldots, a_{2 n+1}\right)^{t}=N^{-1}\left[Y^{n+1}\right]_{\mathcal{S}_{n}(n)}$. Now we have

$$
\begin{aligned}
\beta_{00} & =\left\langle\left[Y^{n+1}\right]_{\tilde{\mathcal{B}}}, X^{n+1}\right\rangle \\
& =a_{1}\left\langle[1]_{\tilde{\mathcal{B}}}, X^{n+1}\right\rangle+a_{2}\left\langle[X]_{\tilde{\mathcal{B}}}, X^{n+1}\right\rangle+\ldots+a_{2 n+1}\left\langle\left[Y^{n}\right]_{\tilde{\mathcal{B}}}, X^{n+1}\right\rangle \\
& =\left[X^{n+1}\right]_{\mathcal{S}_{n}(n)}^{t} \cdot\left(a_{1}, \ldots, a_{2 n+1}\right)^{t} \\
& =\left[X^{n+1}\right]_{\mathcal{S}_{n}(n)}^{t} N^{-1}\left[Y^{n+1}\right]_{\mathcal{S}_{n}(n)}=\eta,
\end{aligned}
$$

a contradiction. Since $a_{2 n+2} \neq 0$, we may now proceed exactly as in the proof of Proposition 2.7 (beginning at (2.5) and replacing $n$ by $n+1$ ) to conclude that $\mathcal{M}(n+1)$ admits a flat extension $\mathcal{M}(n+2)$ (cf. Remark 2.13).

Remark 2.15. Recall that $\mathbf{F}$ depends on $p$ and is independent of $q$, while $\mathbf{G}$ depends on $q$ and is independent of $p$. It follows that $\eta$ is of the form $\eta \equiv \eta(p, q)=$ $a+b p+c q+d p q$, where $a, b, c, d \in \mathbb{R}$ are independent of $p$ and $q$. Thus, if $b, c$ or $d$ is nonzero, it is possible to choose $p$ and $q$ so that $\eta=\beta_{00}$, whence $\mathcal{M}(n)$ admits a flat extension $\mathcal{M}(n+1)$ (and $\beta$ admits a rank $\mathcal{M}(n)$-atomic representing measure). In $\mathrm{CuFi5}$ Proof of Proposition 5.3] we showed that this is the case in the quartic moment problem $(n=2)$, where we always have $b$ or $d$ nonzero. For $n>2$, we do not know whether it is always the case that $b, c$ or $d$ is nonzero.

Proof of Theorem [2.1. Straightforward from Propositions 2.5] 2.6] 2.7] and 2.14]

\section{The Truncated Moment Problem on Degenerate Hyperbolas}

In this section we prove Theorem 1.1 for degenerate hyperbolas. By Proposition 1.9. it suffices to consider the case $y x=0$, and the necessity of the conditions in Theorem 1.1 is clear from Section [1] We establish sufficiency in the following result.

Theorem 3.1. Let $\beta \equiv \beta^{(2 n)}: \beta_{00}, \beta_{01}, \beta_{10}, \ldots, \beta_{0,2 n}, \ldots, \beta_{2 n, 0}$ be a family of real numbers, $\beta_{00}>0$, and let $\mathcal{M}(n)$ be the associated moment matrix. Assume that $\mathcal{M}(n)$ is positive, recursively generated, and satisfies $Y X=0$ and $\operatorname{rank} \mathcal{M}(n) \leq$ card $\mathcal{V}(\beta)$. Then $\operatorname{rank} \mathcal{M}(n) \leq 2 n+1$. If $\operatorname{rank} \mathcal{M}(n) \leq 2 n$, then $\mathcal{M}(n)$ admits a flat extension (so $\beta$ admits a rank $\mathcal{M}(n)$-atomic representing measure supported in $y x=0)$. If $\operatorname{rank} \mathcal{M}(n)=2 n+1$, then $\mathcal{M}(n)$ admits a positive, recursively generated extension $\mathcal{M}(n+1)$, satisfying $2 n+1 \leq \operatorname{rank} \mathcal{M}(n+1) \leq 2 n+2$, and $\mathcal{M}(n+1)$ admits a flat extension $\mathcal{M}(n+2)$ (so $\beta$ admits a representing measure $\mu$ supported in $y x=0$, with $2 n+1 \leq$ card supp $\mu \leq 2 n+2)$.

By $[\mathrm{CuFi2}$, Theorem 2.1], we know that a positive, recursively generated moment matrix $\mathcal{M}(n)$ admits flat extensions when $\{1, X, Y\}$ is linearly dependent in $\mathcal{C}_{\mathcal{M}(n)}$; in the sequel, we therefore assume that $\{1, X, Y\}$ is linearly independent. We begin with an elementary lemma based on recursiveness and $Y X=0$. For $1 \leq k \leq n$ let

$$
\mathcal{S}_{n}(k):=\left\{1, X, Y, X^{2}, Y^{2}, \ldots, X^{k}, Y^{k}\right\} \subseteq \mathcal{C}_{\mathcal{M}(n)}
$$

Lemma 3.2. For $n \geq 2$, let $\mathcal{M}(n)$ be positive and recursively generated, and assume that $Y X=0$. Then each nonzero column of $\mathcal{M}(n)$ is in $\mathcal{S}_{n}(n)$, and therefore $\operatorname{rank} \mathcal{M}(n) \leq 2 n+1$.

In the sequel we also require the following well-known result. 
Lemma 3.3. (Choleski's Algorithm Atk) Let $A$ be a positive and invertible $d \times d$ matrix over $\mathbb{R}$, let $\mathbf{b}$ denote a column vector in $\mathbb{R}^{d}$, and let $c \in \mathbb{R}$. Then $\tilde{A}:=$ $\left(\begin{array}{cc}A & \mathbf{b} \\ \mathbf{b}^{t} & c\end{array}\right)$ is positive and invertible if and only if $\delta:=c-\mathbf{b}^{t} A^{-1} \mathbf{b}>0$. In this
case,

$$
\tilde{A}^{-1}=\frac{1}{\delta}\left(\begin{array}{cc}
\left(\delta+A^{-1} \mathbf{b b}^{t}\right) A^{-1} & -A^{-1} \mathbf{b} \\
-\mathbf{b}^{t} A^{-1} & 1
\end{array}\right) .
$$

We next divide the proof of Theorem 3.1 into three cases, based on possible dependence relations among the elements of $\mathcal{S}_{n}(n)$. Section $[5$ contains examples illustrating these cases. As in Section 2 in each case, once we establish a flat extension, the existence of the required representing measure $\mu$, necessarily supported in $y x=0$, always follows immediately from (1.5) and (1.8), so we will not repeat this argument in each case.

Proposition 3.4. Suppose $\mathcal{M}(n) \equiv \mathcal{M}(n)(\beta)$ is positive, recursively generated, $\operatorname{card} \mathcal{V}(\mathcal{M}(n)) \geq \operatorname{rank} \mathcal{M}(n)$, and $Y X=0$ in $\mathcal{C}_{\mathcal{M}(n)}$. Suppose there exists $k$, $1<k \leq n$, such that $\mathcal{S}_{n}(k-1)$ is linearly independent and $X^{k} \in$ lin.span $\mathcal{S}_{n}(k-1)$. Then $\mathcal{M}(n)$ admits a flat extension $\mathcal{M}(n+1)$ (and $\beta^{(2 n)}$ admits a $\operatorname{rank} \mathcal{M}(n)$ atomic representing measure).

Proof. By hypothesis, we may write

$$
X^{k}=a_{0} 1+a_{1} X+b_{1} Y+\ldots+a_{k-1} X^{k-1}+b_{k-1} Y^{k-1} \quad\left(a_{i}, b_{i} \in \mathbb{R}\right) .
$$

Equation (3.1) implies that there are at most $k$ points in $\mathcal{V}(\mathcal{M}(n))$ of the form $(x, 0)$. If, for some $j, b_{j} \neq 0$, then it follows from (3.1) that there are at most $k-1$ points in $\mathcal{V}(\mathcal{M}(n))$ of the form $(0, y)$. In this case, since $Y X=0$ in $\mathcal{C}_{\mathcal{M}(n)}$, it follows that $2 k-1=\operatorname{card} \mathcal{S}_{n}(k-1) \leq \operatorname{rank} \mathcal{M}(n) \leq \operatorname{card} \mathcal{V}(\mathcal{M}(n)) \leq 2 k-1$, whence $\operatorname{rank} \mathcal{M}(n)=2 k-1$ and $Y^{j} \in$ lin.span $\mathcal{S}_{n}(k-1)(k \leq j \leq n)$. If $k=n$, this shows that $\mathcal{M}(n)$ is flat. If $k<n$, then also $X^{j} \in \operatorname{lin}$.span $\mathcal{S}_{n}(k-1)(k+1 \leq j \leq n)$, so again $\mathcal{M}(n)$ is flat. Thus, if some $b_{j} \neq 0$, then $\mathcal{M}(n)$ is flat and the result follows. We may thus assume that each $b_{j}=0$, i.e.,

$$
X^{k}=a_{0} 1+a_{1} X+\ldots+a_{k-1} X^{k-1} .
$$

If $a_{0} \neq 0$, there are no points in $\mathcal{V}(\mathcal{M}(n))$ of the form $(0, y)$. Thus, in this case, each point in the variety is of the form $(x, 0)$, and (3.2) implies that there can be at most $k$ such points. Then $2 k-1 \leq \operatorname{rank} \mathcal{M}(n) \leq \operatorname{card} \mathcal{V}(\mathcal{M}(n)) \leq k$, a contradiction. We thus conclude that $a_{0}=0$, whence

$$
X^{k}=a_{1} X+\ldots+a_{k-1} X^{k-1} .
$$

By recursiveness,

$$
X^{k+i}=a_{1} X^{i+1}+\ldots+a_{k-1} X^{k-1+i} \quad(1 \leq i \leq n-k) .
$$

Thus $\tilde{\mathcal{B}}:=\left\{1, X, Y, \ldots, X^{k-1}, Y^{k-1}, Y^{k}, \ldots, Y^{n}\right\}$ spans $\mathcal{C}_{\mathcal{M}(n)}$. If $\tilde{\mathcal{B}}$ is linearly dependent, it follows readily from recursiveness and $Y X=0$ (as above) that $\mathcal{M}(n)$ is flat, so again there is a (unique, $\operatorname{rank} \mathcal{M}(n)$-atomic) representing measure.

We may thus assume that $\tilde{\mathcal{B}}$ is a basis for $\mathcal{C}_{\mathcal{M}(n)}$. We will show that $\mathcal{M}(n)$ admits infinitely many flat extensions $\mathcal{M}(n+1)$ (each corresponding to a distinct $\operatorname{rank} \mathcal{M}(n)$-atomic representing measure). To define

$$
\mathcal{M}(n+1) \equiv\left(\begin{array}{cc}
\mathcal{M}(n) & B(n+1) \\
B(n+1)^{t} & C(n+1)
\end{array}\right)
$$


which must be recursively generated (since $\mathcal{M}(n+1)$ would have a representing measure by (1.8)), we first use (3.3) and (3.4) to define $X^{n+1}$ in $\mathcal{C}_{(\mathcal{M}(n) B(n+1))}$ by

$$
X^{n+1}:=a_{1} X^{n+2-k}+\ldots+a_{k-1} X^{n} .
$$

Clearly, $X^{n+1} \in \operatorname{Ran} \mathcal{M}(n)$. To ensure the moment matrix structure of $X^{n+1}$, we must verify that

$$
\left\langle X^{n+1}, Y^{i} X^{j}\right\rangle=\beta_{i, n+1+j}(0 \leq i+j \leq n-1) .
$$

If $i>0$, then $\left\langle X^{p}, Y^{i} X^{j}\right\rangle=0 \quad(0<p \leq n)$, so (3.5) implies $\left\langle X^{n+1}, Y^{i} X^{j}\right\rangle=0=$ $\beta_{i, n+1+j}$ in this case. For $i=0$,

$$
\begin{aligned}
\left\langle X^{n+1}, X^{j}\right\rangle & =\sum_{s=1}^{k-1} a_{s}\left\langle X^{n+1-k+s}, X^{j}\right\rangle=\sum_{s=1}^{k-1} a_{s}\left\langle X^{n-k+s}, X^{j+1}\right\rangle \\
& =\left\langle X^{n}, X^{j+1}\right\rangle \quad(\text { by }(\text { 3.4) }) \\
& =\beta_{0, n+1+j} \quad(\text { since } 0 \leq j \leq n-1) .
\end{aligned}
$$

We next define $Y^{n+1}$ in $\mathcal{C}_{(\mathcal{M}(n) B(n+1))}$ consistent with moment matrix structure and $Y X=0 . \quad$ Let $\mathcal{B}:=\left\{1, X, \ldots, X^{k-1}\right\} \bigcup\left\{Y, \ldots,, Y^{k-1}, \ldots, Y^{n-1}\right\} \subseteq \mathcal{C}_{\mathcal{M}(n)}$; $\left[Y^{n+1}\right]_{\mathcal{B}}$ is uniquely determined by $\left\langle Y^{n+1}, X^{j}\right\rangle=0(1 \leq j \leq k-1)$ and $\left\langle Y^{n+1}, Y^{i}\right\rangle=$ $\beta_{n+1+i, 0}(0 \leq i \leq n-1)$. Now

$$
\left[Y^{n+1}\right]_{\tilde{\mathcal{B}}}=\left(\begin{array}{c}
{\left[Y^{n+1}\right]_{\mathcal{B}}} \\
q
\end{array}\right)(q \in \mathbb{R}),
$$

and every other component of $Y^{n+1}$ (besides those in $\left[Y^{n+1}\right]_{\tilde{\mathcal{B}}}$ ) must be zero (corresponding to $\left\langle Y^{n+1}, Y^{r} X^{s}\right\rangle$ for some $\left.s>0\right)$.

We will show that for each $q \in \mathbb{R}, Y^{n+1} \in \operatorname{Ran} \mathcal{M}(n)$. Let $\mathcal{M}:=[\mathcal{M}(n)]_{\mathcal{B}}>0$. For each vector of the form $[v]_{\mathcal{B}}, \mathcal{M}^{-1}\left([v]_{\mathcal{B}}\right) \equiv\left(r_{0}, r_{1}, s_{1}, \ldots, r_{k-1}, s_{k-1}, \ldots, s_{n-1}\right)$ uniquely satisfies

$[v]_{\mathcal{B}}=r_{0}[1]_{\mathcal{B}}+r_{1}[X]_{\mathcal{B}}+s_{1}[Y]_{\mathcal{B}}+\ldots+r_{k-1}\left[X^{k-1}\right]_{\mathcal{B}}+s_{k-1}\left[Y^{k-1}\right]_{\mathcal{B}}+\ldots+s_{n-1}\left[Y^{n-1}\right]_{\mathcal{B}}$

We claim that

$$
\left[X^{k+j}\right]_{\mathcal{B}}^{t} \mathcal{M}^{-1}\left[Y^{n}\right]_{\mathcal{B}}=0(0 \leq j \leq n-k) .
$$

Indeed, (3.3) and (3.4) together imply that $X^{k+j}$ is a linear combination of $X$, $X^{2}, \ldots, X^{k-1}$, so in (3.8), all the coordinates of $\mathcal{M}^{-1}\left[X^{k+j}\right]_{\mathcal{B}}$ corresponding to $1, Y, \ldots, Y^{n-1}$ equal zero. By the $Y X=0$ structure of $\mathcal{M}(n)$, the only coordinates of $\left[Y^{n}\right]_{\mathcal{B}}$ that are possibly nonzero correspond to $1, Y, \ldots, Y^{n-1}$. These observations show that $\left[Y^{n}\right]_{\mathcal{B}}^{t} \mathcal{M}^{-1}\left[X^{k+j}\right]_{\mathcal{B}}=0$, whence (3.9) follows. Similarly, the $Y X=0$ structure of $Y^{n+1}$ implies that

$$
\left[X^{k+j}\right]_{\mathcal{B}}^{t} \mathcal{M}^{-1}\left[Y^{n+1}\right]_{\mathcal{B}}=0(0 \leq j \leq n-k)
$$

Since $\tilde{\mathcal{M}}:=[\mathcal{M}(n)]_{\tilde{\mathcal{B}}}>0$, we have $\left[Y^{n+1}\right]_{\tilde{\mathcal{B}}} \in \operatorname{Ran} \tilde{\mathcal{M}}$. To show that $Y^{n+1} \in \operatorname{Ran}$ $\mathcal{M}(n)$, the $Y X=0$ structure of $\mathcal{M}(n)$ and of $Y^{n+1}$ imply that it suffices to verify that

$$
\left[X^{k+j}\right]_{\tilde{\mathcal{B}}}^{t}(\tilde{\mathcal{M}})^{-1}\left[Y^{n+1}\right]_{\tilde{\mathcal{B}}}=0(0 \leq j \leq n-k)
$$


Now $\tilde{\mathcal{M}}=\left(\begin{array}{cc}\mathcal{M} & {\left[Y^{n}\right]_{\mathcal{B}}} \\ {\left[Y^{n}\right]_{\mathcal{B}}^{t}} & c\end{array}\right) \equiv\left(\begin{array}{cc}\mathcal{M} & \mathbf{b} \\ \mathbf{b}^{t} & c\end{array}\right)$, so

$$
(\tilde{\mathcal{M}})^{-1}=\frac{1}{\delta}\left(\begin{array}{cc}
\left(\delta+\mathcal{M}^{-1} \mathbf{b} \mathbf{b}^{t}\right) \mathcal{M}^{-1} & -\mathcal{M}^{-1} \mathbf{b} \\
-\mathbf{b}^{t} \mathcal{M}^{-1} & 1
\end{array}\right),
$$

with $\delta:=c-\mathbf{b}^{t} \mathcal{M}^{-1} \mathbf{b}$, by Lemma 3.3 We also have $\left[X^{k+j}\right]_{\tilde{\mathcal{B}}}^{t}=\left(\left[X^{k+j}\right]_{\mathcal{B}}^{t} 0\right)$ (since $\left\langle X^{k+j}, Y^{n}\right\rangle=0$ ); thus, using (3.7), we have

$$
\begin{aligned}
{\left[X^{k+j}\right]_{\tilde{\mathcal{B}}}^{t} \tilde{\mathcal{M}}^{-1}\left[Y^{n+1}\right]_{\tilde{\mathcal{B}}} } & =\frac{1}{\delta}\left[X^{k+j}\right]_{\mathcal{B}}^{t}\left(\left(\delta+\mathcal{M}^{-1} \mathbf{b b}^{t}\right) \mathcal{M}^{-1}\left[Y^{n+1}\right]_{\mathcal{B}}-q \mathcal{M}^{-1} \mathbf{b}\right) \\
& =\left[X^{k+j}\right]_{\mathcal{B}}^{t} \mathcal{M}^{-1}\left[Y^{n+1}\right]_{\mathcal{B}} \\
& +\frac{1}{\delta}\left(\left[X^{k+j}\right]_{\mathcal{B}}^{t} \mathcal{M}^{-1} \mathbf{b}\right) \mathbf{b}^{t} \mathcal{M}^{-1}\left[Y^{n+1}\right]_{\mathcal{B}} \\
& -\frac{q}{\delta}\left[X^{k+j}\right]_{\mathcal{B}}^{t} \mathcal{M}^{-1} \mathbf{b} \\
& =0 \text { (by (3.9) and (3.10). }
\end{aligned}
$$

Thus $Y^{n+1} \in \operatorname{Ran} \mathcal{M}(n)$.

We now have columns $X^{n+1}$ and $Y^{n+1}$ for block $B(n+1)$, and to preserve the $Y X=0$ structure we set $Y^{i} X^{j}=0(i+j=n+1 ; i, j>0)$. Thus $\operatorname{Ran} B(n+1) \subseteq$ Ran $\mathcal{M}(n)$, and we consider the flat extension

$$
\hat{\mathcal{M}}:=[\mathcal{M}(n) ; B(n+1)] \equiv\left(\begin{array}{cc}
\mathcal{M}(n) & B(n+1) \\
B(n+1)^{t} & C
\end{array}\right) .
$$

To complete the proof, we will show that $C$ has the form of a moment matrix block consistent with $Y X=0$. To do so, from (3.12) and the $Y X=0$ structures of $\mathcal{M}(n)$ and $B(n+1)$, it suffices to show that $\left\langle X^{n+1}, Y^{n+1}\right\rangle=0$. Now,

$$
\begin{aligned}
& \left.\left\langle X^{n+1}, Y^{n+1}\right\rangle=\sum_{j=1}^{k-1} a_{j}\left\langle X^{n+1-k+j}, Y^{n+1}\right\rangle \quad \text { by (3.5) and (3.12) }\right) \\
& =\sum_{j=1}^{k-1} a_{j}\left\langle Y^{n+1}, X^{n+1-k+j}\right\rangle \quad\left(\text { since } \hat{\mathcal{M}}=\left(\mathcal{M}^{\hat{}}\right)^{t}\right) \\
& =0 \text { (since } Y^{n+1} \text { in } B(n+1) \text { has } Y X=0 \text { structure). }
\end{aligned}
$$

Thus $\mathcal{M}$ is a flat moment matrix extension of $\mathcal{M}(n)$; the proof is complete.

We next consider the case when the first column dependence relation occurs at $Y^{k}(1<k \leq n)$.

Proposition 3.5. Assume $\mathcal{M}(n)(\beta)$ is positive, recursively generated, and $Y X=0$ in $\mathcal{C}_{\mathcal{M}(n)}$. Suppose that for some $k(1<k \leq n), \mathcal{S} \equiv\left\{1, X, Y, \ldots, X^{k-1}, Y^{k-1}, X^{k}\right\}$ is linearly independent and $Y^{k} \in$ lin.span $\mathcal{S}$. Then $\mathcal{M}(n)$ admits a flat extension (and $\beta^{(2 n)}$ admits a $\operatorname{rank} \mathcal{M}(n)$-atomic representing measure).

Proof. In $\mathcal{C}_{\mathcal{M}(n)}$ we have a dependence relation

$$
Y^{k}=a_{0} 1+a_{1} X+b_{1} Y+\ldots+a_{k-1} X^{k-1}+b_{k-1} Y^{k-1}+a_{k} X^{k} .
$$

If $a_{k}=0$, we can interchange the roles of $X$ and $Y$ and invoke Proposition 3.4. We may thus assume $a_{k} \neq 0$, so there is a dependence relation of the form

$$
X^{k}=\tilde{a}_{0} 1+\tilde{a}_{1} X+\tilde{b}_{1} Y+\ldots+\tilde{a}_{k-1} X^{k-1}+\tilde{b}_{k-1} Y^{k-1}+\tilde{b}_{k} Y^{k} .
$$


Since any flat extension $\mathcal{M}(n+1)$ must be recursively generated, with $Y X=0$, in $B(n+1)$ we must have

$$
Y^{n+1}=a_{0} Y^{n+1-k}+b_{1} Y^{n+2-k}+\ldots+b_{k-1} Y^{n}
$$

and

$$
X^{n+1}=\tilde{a}_{0} X^{n+1-k}+\tilde{a}_{1} X^{n+2-k}+\ldots+\tilde{a}_{k-1} X^{n}
$$

let $\mathbf{w}$ denote the column vector of length $m(n)$ such that

$$
\left\langle\mathbf{w}, Y^{i} X^{j}\right\rangle:=\left\{\begin{array}{l}
\tilde{a}_{j-n-1+k} \text { if } n+1-k \leq j \leq n \text { and } i=0 \\
0 \text { otherwise }
\end{array} .\right.
$$

To show that $Y^{n+1}$ is consistent with moment matrix structure and $Y X=0$, we first verify that

$$
\left\langle Y^{n+1}, Y^{i} X^{j}\right\rangle=\beta_{n+1+i, j}(0 \leq i+j \leq n-1)
$$

indeed,

$$
\begin{aligned}
\left\langle Y^{n+1}, Y^{i} X^{j}\right\rangle & =\left\langle a_{0} Y^{n+1-k}, Y^{i} X^{j}\right\rangle+\left\langle b_{1} Y^{n+2-k}, Y^{i} X^{j}\right\rangle+\ldots+\left\langle b_{k-1} Y^{n}, Y^{i} X^{j}\right\rangle \\
& =\left\langle a_{0} Y^{n-k}, Y^{i+1} X^{j}\right\rangle+\left\langle b_{1} Y^{n+1-k}, Y^{i+1} X^{j}\right\rangle+\ldots \\
& +\left\langle b_{k-1} Y^{n-1}, Y^{i+1} X^{j}\right\rangle \\
& =\left\langle Y^{n}, Y^{i+1} X^{j}\right\rangle \text { (by recursiveness, using (3.13) } \\
& \left.=\beta_{n+i+1, j} \text { (by the structure of } \mathcal{M}(n)\right) .
\end{aligned}
$$

Since (3.15) readily implies that $\left\langle Y^{n+1}, Y^{i} X^{j}\right\rangle=0$ when $j>0$ and $i+j=n$, it follows that $Y^{n+1}$ is consistent. A similar argument (using (3.14) and (3.16) ) shows that $X^{n+1}$ is also consistent with moment matrix structure and $Y X=0$. Now, setting $Y^{i} X^{j}=0$ in $B(n+1)$ for $i+j=n+1(i, j>0)$, we have a moment matrix block $B(n+1)$ consistent with $Y X=0$ and satisfying $\operatorname{Ran} B(n+1) \subseteq \operatorname{Ran} \mathcal{M}(n)$. Consider $\mathcal{M}^{\wedge}:=[\mathcal{M}(n) ; B(n+1)] \equiv\left(\begin{array}{cc}\mathcal{M}(n) & B(n+1) \\ B(n+1)^{t} & C\end{array}\right)$. To show that $\mathcal{M}^{\wedge}$ is a moment matrix, it suffices to check that in block $C, C_{n+2,1} \equiv\left\langle X^{n+1}, Y^{n+1}\right\rangle=0$, and this follows immediately from the identity $C_{n+2,1}=\left[Y^{n+1}\right]_{m(n)}^{t} \cdot \mathbf{w}$.

The following result concludes the proof of Theorem 3.1 .

Proposition 3.6. Assume that $\mathcal{M} \equiv \mathcal{M}(n)$ is positive, recursively generated, satisfies $Y X=0$, and that $\mathcal{S}_{n}(n)$ is a basis for $\mathcal{C}_{\mathcal{M}(n)}$. Then either $\mathcal{M}(n)$ admits a flat extension (and $\beta$ admits a $(2 n+1)$-atomic representing measure) or $\mathcal{M}(n)$ admits a rank- $(2 n+2)$ positive, recursively generated extension $\mathcal{M}(n+1)$ which has a flat extension $\mathcal{M}(n+2)$ (and $\beta$ admits a $(2 n+2)$-atomic representing measure).

Proof. By hypothesis, $\mathcal{B}:=\left\{1, X, Y, \ldots, X^{n}, Y^{n}\right\}$ is a basis for $\mathcal{S}_{n}(n)$, so the compression $A \equiv[\mathcal{M}]_{\mathcal{B}}$ of $\mathcal{M}$ to the rows and columns of $\mathcal{B}$ is positive and invertible. We define the columns $X^{n+1}$ and $Y^{n+1}$ in the proposed block $B(n+1)$ by

$$
\begin{aligned}
\left\langle X^{n+1}, X^{i}\right\rangle & :=\left\{\begin{array}{cc}
\left\langle X^{n}, X^{i+1}\right\rangle\left(=\beta_{0, n+i+1}\right) & 0 \leq i<n \\
p & i=n
\end{array}\right. \\
\left\langle Y^{n+1}, Y^{j}\right\rangle & :=\left\{\begin{array}{cc}
\left\langle Y^{n}, Y^{j+1}\right\rangle\left(=\beta_{n+j+1,0}\right) & 0 \leq j<n \\
q & j=n
\end{array}\right.
\end{aligned}
$$

and $\left\langle X^{n+1}, Y^{j} X^{i}\right\rangle:=0(1 \leq i+j \leq n ; j \geq 1)$ and $\left\langle Y^{n+1}, Y^{j} X^{i}\right\rangle:=0(1 \leq$ $i+j \leq n ; i \geq 1)$, where $p$ and $q$ are two parameters. Let $\mathbf{r}_{p}:=\left[X^{n+1}\right]_{\mathcal{B}}$ and 
$\mathbf{s}_{q}:=\left[Y^{n+1}\right]_{\mathcal{B}}$. Due to the $Y X=0$ structure of $\mathcal{M}(n)$, it is straightforward to check that $\operatorname{Ran} B(n+1) \subseteq \operatorname{Ran} \mathcal{M}$, and that if $W$ is a matrix satisfying $\mathcal{M} W=$ $B(n+1)$, then

$$
C \equiv W^{*} \mathcal{M} W=\left(\begin{array}{ccccc}
\mathbf{r}_{p}^{t} A^{-1} \mathbf{r}_{p} & 0 & \cdots & 0 & \mathbf{r}_{p}^{t} A^{-1} \mathbf{s}_{q} \\
0 & 0 & \cdots & 0 & 0 \\
\vdots & \vdots & \ddots & \vdots & \vdots \\
0 & 0 & \cdots & 0 & 0 \\
\mathbf{s}_{q}^{t} A^{-1} \mathbf{r}_{p} & 0 & \cdots & 0 & \mathbf{s}_{q}^{t} A^{-1} \mathbf{s}_{q}
\end{array}\right)
$$

It follows at once that $\mathcal{M}$ admits a flat extension $\mathcal{M}(n+1)$ if and only if there exist real numbers $p$ and $q$ such that

$$
\alpha(p, q):=\mathbf{s}_{q}^{t} A^{-1} \mathbf{r}_{p}=0 .
$$

We may thus assume that $\alpha$ is nonzero on $\mathbb{R}^{2}$. Fix $p, q \in \mathbb{R}$ and let $u>\mathbf{r}_{p}^{t} A^{-1} \mathbf{r}_{p}$ and $v:=\frac{\alpha^{2}}{u-\mathbf{r}_{p}^{t} A^{-1} \mathbf{r}_{p}}+\mathbf{s}_{q}^{t} A^{-1} \mathbf{s}_{q}$, so that

$$
C(u, v):=\left(\begin{array}{ccccc}
u & 0 & \cdots & 0 & 0 \\
0 & 0 & \cdots & 0 & 0 \\
\vdots & \vdots & \ddots & \vdots & \vdots \\
0 & 0 & \cdots & 0 & 0 \\
0 & 0 & \cdots & 0 & v
\end{array}\right) \geq C(n+1)
$$

and

$$
\operatorname{rank}(C(u, v)-C)=1
$$

Then

$$
\mathcal{M}(n+1) \equiv \mathcal{M}(n+1 ; u, p, q):=\left(\begin{array}{cc}
\mathcal{M} & B(n+1) \\
B(n+1)^{*} & C(u, v)
\end{array}\right)
$$

is positive and recursively generated, and $\operatorname{rank} \mathcal{M}(n+1)=1+\operatorname{rank} \mathcal{M}(=2 n+2)$.

We claim that $\mathcal{M}(n+1)$ admits a flat extension $\mathcal{M}(n+2)$. We first show that there is a unique block $B(n+2)$, subordinate to $Y X=0$, such that Ran $B(n+2) \subseteq \operatorname{Ran} \mathcal{M}(n+1)$. In any such block, $Y X^{n+1}=Y^{2} X^{n}=\ldots=Y^{n+1} X=0$. In $Y^{n+2}$, all the entries are determined from $\mathcal{M}(n+1)$ and $Y X=0$, except $s:=\left\langle Y^{n+2}, Y^{n+1}\right\rangle$. Now, in $\mathcal{C}_{\mathcal{M}(n+1)}$ we have a dependence relation

$$
Y^{n+1}=p_{n+1}(X)+q_{n}(Y)
$$

with $\operatorname{deg} p_{n+1} \leq n+1, \operatorname{deg} q_{n} \leq n$. Since a flat extension must necessarily be recursively generated, in $\mathcal{C}_{\mathcal{M}(n+2)}$ we must have

$$
Y^{n+2}=p_{n+1}(0) Y+Y q_{n}(Y),
$$

whence $s=\left\langle p_{n+1}(0) Y+Y q_{n}(Y), Y^{n+1}\right\rangle$. Thus, $Y^{n+2} \in \operatorname{Ran} \mathcal{M}(n+1)$ and $Y^{n+2}$ has $Y X=0$ structure and is Hankel with respect to $Y^{n+1} X$.

Note that if $X^{n+2}$ for block $B(n+2)$ is defined to be consistent with known moment values and $Y X=0$ structure, then every value in $X^{n+2}$ is determined except $r:=\left\langle X^{n+2}, X^{n+1}\right\rangle$. We next show that there is a unique value of $r$ such that $X^{n+2} \in \operatorname{Ran} \mathcal{M}(n+1)$. Since $u>\mathbf{r}_{p}^{t} A^{-1} \mathbf{r}_{p}^{t}$, Smul'jan's Theorem [Smu] (described in Section 1) implies that the compression $\tilde{A}$ of $\mathcal{M}(n+1)$ to rows and 
columns given by $\tilde{\mathcal{B}}:=\mathcal{B} \bigcup\left\{X^{n+1}\right\}$ is positive and invertible, and of the form $\tilde{A}=\left(\begin{array}{cc}A & \mathbf{r}_{p} \\ \mathbf{r}_{p}^{t} & u\end{array}\right)$. We now apply Lemma 3.3 to obtain

$$
\tilde{A}^{-1}=\frac{1}{\delta}\left(\begin{array}{cc}
\left(\delta+A^{-1} \mathbf{r}_{p} \mathbf{r}_{p}^{t}\right) A^{-1} & -A^{-1} \mathbf{r}_{p} \\
-\mathbf{r}_{p}^{t} A^{-1} & 1
\end{array}\right) \quad\left(\delta:=u-\mathbf{r}_{p}^{t} A^{-1} \mathbf{r}_{p}\right)
$$

Observe that $\left[X^{n+2}\right]_{\tilde{\mathcal{B}}} \equiv\left(\begin{array}{c}{\left[X^{n+2}\right]_{\mathcal{B}}} \\ r\end{array}\right)$, and that, apart from the entries in $\left[X^{n+2}\right]_{\tilde{\mathcal{B}}}$, all other entries of $X^{n+2}$ in $B(n+2)$ are zero. From the $Y X=0$ structure of $\mathcal{M}(n)$, it follows that $X^{n+2} \in \operatorname{Ran} \mathcal{M}(n+1)$ if and only if

$$
\left\langle X^{n+2}, Y^{n+1}\right\rangle:=\left[Y^{n+1}\right]_{\tilde{\mathcal{B}}}^{t} \tilde{A}^{-1}\left[X^{n+2}\right]_{\tilde{\mathcal{B}}}=0,
$$

so it suffices to show that (3.19) admits a unique solution for $r$. Now,

$$
\begin{aligned}
& {\left[Y^{n+1}\right]_{\tilde{\mathcal{B}}}^{t} \tilde{A}^{-1}\left[X^{n+2}\right]_{\tilde{\mathcal{B}}}=\left(\begin{array}{cc}
\mathbf{s}_{q}^{t} & 0
\end{array}\right)\left(\begin{array}{cc}
A & \mathbf{r}_{p} \\
\mathbf{r}_{p}^{t} & u
\end{array}\right)^{-1}\left(\begin{array}{c}
{\left[X^{n+2}\right]_{\mathcal{B}}} \\
r
\end{array}\right)} \\
& =\left(\begin{array}{ll}
\mathbf{s}_{q}^{t} & 0
\end{array}\right) \frac{1}{\delta}\left(\begin{array}{cc}
\left(\delta+A^{-1} \mathbf{r}_{p} \mathbf{r}_{p}^{t}\right) A^{-1} & -A^{-1} \mathbf{r}_{p} \\
-\mathbf{r}_{p}^{t} A^{-1} & 1
\end{array}\right)\left(\begin{array}{c}
{\left[X^{n+2}\right]_{\mathcal{B}}} \\
r
\end{array}\right) \\
& =\frac{1}{\delta}\left(\begin{array}{ll}
\mathbf{s}_{q}^{t} & 0
\end{array}\right)\left(\begin{array}{c}
H-r A^{-1} \mathbf{r}_{p} \\
*
\end{array}\right) \quad(\text { for a certain vector } H) \\
& =\frac{1}{\delta}\left(\mathbf{s}_{q}^{t} H-r \mathbf{s}_{q}^{t} A^{-1} \mathbf{r}_{p}\right)=\frac{1}{\delta}\left(\mathbf{s}_{q}^{t} H-r \alpha(p, q)\right) .
\end{aligned}
$$

Since $\alpha(p, q) \neq 0$, it follows that (3.19) admits a unique solution $r \equiv r(p, q, u)$.

With this value, $\operatorname{Ran} B(n+1) \subseteq \operatorname{Ran} \mathcal{M}(n+1)$, so $B(n+2)=\mathcal{M}(n+1) W$ for some matrix $W$. To show that the flat extension $\mathcal{M}_{n+2}:=[\mathcal{M}(n+1) ; B(n+2)] \equiv$ $\left(\begin{array}{cc}\mathcal{M}(n+1) & B(n+2) \\ B(n+2)^{t} & C_{n+2}\end{array}\right)$ is a moment matrix, it now suffices to show that $\left\langle Y^{n+2}, X^{n+2}\right\rangle=0$; this is because, by positivity of $\mathcal{M}_{n+2},\left\langle X^{n+2}, Y^{n+2}\right\rangle=$ $\left\langle Y^{n+2}, X^{n+2}\right\rangle$ and, by flatness, all other entries of $C_{n+2}$ (except $\left\langle X^{n+2}, X^{n+2}\right\rangle$ and $\left.\left\langle Y^{n+2}, Y^{n+2}\right\rangle\right)$ are clearly zero. Now recall that $Y^{n+2}=p_{n+1}(0) Y+Y q_{n}(Y)$ in $\mathcal{C}_{(\mathcal{M}(n+1) B(n+2))}$ (by (3.18)), so by flatness, the same relation must hold in $\mathcal{C}_{\mathcal{M}_{n+2}}$. Thus,

$\left\langle Y^{n+2}, X^{n+2}\right\rangle=\left\langle p_{n+1}(0) Y+Y q_{n}(Y), X^{n+2}\right\rangle=\left\langle X^{n+2}, p_{n+1}(0) Y+Y q_{n}(Y)\right\rangle=0$, since, by the construction of $X^{n+2}$ in $B(n+2)$ (consistent with the relation $Y X=$ $0),\left\langle X^{n+2}, Y^{j+1}\right\rangle=0(0 \leq j \leq n)$.

Remark 3.7. Example[5.4 (below) illustrates a case of Proposition 3.6 where $\mathcal{M}(2)$ admits no flat extension, so the minimal representing measure is $(2 n+2)$-atomic.

\section{Proof of Theorem 1.5}

We now turn to the proof of Theorem [1.5 which we restate for the sake of convenience. As in previous sections, it suffices to consider the cases $y x=1$ and $y x=0$.

Theorem 4.1. Let $\beta \equiv \beta^{(2 n)}: \beta_{00}, \beta_{01}, \beta_{10}, \ldots, \beta_{0,2 n}, \ldots, \beta_{2 n, 0}$ be a family of real numbers, $\beta_{00}>0$, and let $\mathcal{M}(n)$ be the associated moment matrix. Assume that $\mathcal{M}(n)$ is positive, recursively generated, and satisfies $Y X=1$ (resp. $Y X=0$ ). Then $\operatorname{rank} \mathcal{M}(n) \leq 2 n+1$, and the following statements are equivalent. 
(i) $\beta$ admits a representing measure (necessarily supported in $y x=1$, resp. $y x=0)$.

(ii) $\beta$ admits a representing measure with convergent moments up to degree $2 n+2$ (necessarily supported in $y x=1$, resp. $y x=0$ ).

(iii) $\beta$ admits a representing measure $\mu$ (necessarily supported in $y x=1$, resp. $y x=0)$ such that card $\operatorname{supp} \mu \leq 1+\operatorname{rank} M(n)$. If $\operatorname{rank} M(n) \leq 2 n$, then $\mu$ can be taken so that card $\operatorname{supp} \mu=\operatorname{rank} M(n)$.

(iv) $M(n)$ admits a positive, recursively generated extension $M(n+1)$.

(v) $M(n)$ admits a positive, recursively generated extension $M(n+1)$, with $\operatorname{rank} M(n+1) \leq 1+\operatorname{rank} M(n)$, and $M(n+1)$ admits a flat extension $M(n+2)$. If rank $M(n) \leq 2 n$, then $M(n)$ admits a flat extension $M(n+1)$.

(vi) $\operatorname{rank} M(n) \leq \operatorname{card} V(\beta)$.

To establish Theorem 4.1 we require the following result, whose proof is an adaptation of the proof of Proposition 3.4

Proposition 4.2. Suppose $\mathcal{M}(n) \equiv \mathcal{M}(n)(\beta)$ is positive, $Y X=0$ in $\mathcal{C}_{\mathcal{M}(n)}$, and $\mathcal{M}(n)$ has a positive, recursively generated extension $\mathcal{M}(n+1)$. Suppose also that there exists $k, 1<k \leq n$, such that $\mathcal{S}_{n}(k-1)$ is linearly independent and $X^{k} \in$ lin.span $\mathcal{S}_{n}(k-1)$. Then $\mathcal{M}(n)$ admits a flat extension $\mathcal{M}(n+1)$.

Proof. By hypothesis, we may write

$$
X^{k}=a_{0} 1+a_{1} X+b_{1} Y+\ldots+a_{k-1} X^{k-1}+b_{k-1} Y^{k-1} \quad\left(a_{i}, b_{i} \in \mathbb{R}\right) .
$$

Assume first that not all coefficients $b_{j}$ are zero, and let $m \leq k-1$ be the largest integer such that $b_{m} \neq 0$. In $\mathcal{M}(n+1)$ we can formally multiply (4.1) by $Y$ to obtain

$$
0=Y X^{k}=a_{0} Y+b_{1} Y^{2}+\ldots+b_{m} Y^{m+1}
$$

from which it follows that $Y^{m+1}$ is a linear combination of columns associated to powers of $y$ of lower degree, and, a fortiori, that the same is true of $Y^{n}$. If we instead formally multiply (4.1) by $X$, we see that $X^{n}$ is a linear combination of columns associated to powers of $x$ of lower degree. Thus, $\mathcal{M}(n)$ is flat. Since $\mathcal{M}(n+1)$ is a recursively generated extension of $\mathcal{M}(n)$, it must be a flat extension of $\mathcal{M}(n)$, and the result follows in this case.

We can thus assume that all coefficients $b_{j}$ are zero, that is,

$$
X^{k}=a_{0} 1+a_{1} X+\ldots+a_{k-1} X^{k-1} .
$$

If $a_{0} \neq 0$, in $\mathcal{M}(n+1)$ we can formally multiply (4.2) by $Y$ to obtain $Y=0$, a contradiction. Thus, (4.2) does not involve the column 1, just as in (3.3). We may now continue exactly as in the proof of Proposition 3.4 since the part of that proof following (3.3) does not entail the variety condition $\operatorname{rank} \mathcal{M}(n) \leq \operatorname{card} \mathcal{V}(\beta)$.

Proof of Theorem 4.1. By CuFi2, Theorem 2.1] and the equivalence of the moment problems for $M(n)(\gamma)$ and $\mathcal{M}(n)(\beta)$ CuFi6 Proposition 1.12], we can assume that the columns $1, X$ and $Y$ are linearly independent. Observe first that $($ iii $) \Rightarrow$ $(i i) \Rightarrow(i)$ trivially, that $(i) \Rightarrow(v i)$ by $\mathrm{CuFi3}$ (1.7)], and that $(i i i) \Leftrightarrow(v)$ by (1.8) and (1.5). Also, $(v i) \Rightarrow(i i i)$ by Theorem 1.1] so $(i),(i i),(i i i),(v)$ and $(v i)$ are equivalent. Since $(v) \Rightarrow(i v)$ is trivial, to complete the proof it suffices to establish $(i v) \Rightarrow(v)$.

Assume first that $\mathcal{M}(n)$ satisfies $Y X=1$. The cases when $\operatorname{rank} \mathcal{M}(n) \leq 2 n$ correspond to the column dependence relations that we considered in Cases I-III in the proof of Theorem [2.1] so we reconsider these dependence relations. First 
recall our hypotheses: $\mathcal{M}(n)$ is positive, recursively generated, $Y X=1$, and $\mathcal{M}(n)$ admits a positive recursively generated extension $\mathcal{M}(n+1)$. We need to show that $\mathcal{M}(n)$ admits a flat extension.

Case I. We have $\mathcal{S}_{n}(k-1)$ linearly independent and $X^{k}=p_{k-1}(X)+q_{k-1}(Y)$ in $\mathcal{C}_{\mathcal{M}(n)}$, with $\operatorname{deg} p_{k-1}, \operatorname{deg} q_{k-1} \leq k-1$. By the Extension Principle [Fia1], the same relation must hold in the column space of the positive extension $\mathcal{M}(n+1)$. Since $\mathcal{M}(n+1)$ is recursively generated, we must also have

$$
X^{k-1} \equiv Y X^{k}=Y p_{k-1}(X)+Y q_{k-1}(Y) .
$$

Let $b_{k-1}$ be the coefficient of $y^{k-1}$ in $q_{k-1}$. If $b_{k-1}=0$, then (4.3) implies that $\mathcal{S}_{n}(k-1)$ is linearly dependent, a contradiction. Thus, $b_{k-1} \neq 0$, so (4.3) implies that $Y^{k}$ can be written as a linear combination of previous columns, and therefore $\mathcal{M}(k)$ is flat; it now follows from Fia1 and recursiveness that $\mathcal{M}(n)$ is flat, and thus admits a flat extension.

Case II. Suppose $\mathcal{S}:=\mathcal{S}_{n}(k-1) \bigcup\left\{X^{k}\right\}$ is linearly independent and $Y^{k} \in$ lin.span $\mathcal{S}$, for some $k<n$. The hypothesis about $\mathcal{M}(n+1)$ is superfluous, as we showed in Proposition 2.6 that $\mathcal{M}(n)$ is flat.

Case III. Here $\mathcal{S}_{n}(n-1) \bigcup\left\{X^{n}\right\}$ is linearly independent and

$$
Y^{n}=a_{n} X^{n}+p_{n-1}(X)+q_{n-1}(Y)
$$

in $\mathcal{C}_{\mathcal{M}(n)}$, with $\operatorname{deg} p_{n-1}, \operatorname{deg} q_{n-1} \leq n-1$. The Extension Principle [Fia1] shows that the same relation must hold in the column space of the positive extension $\mathcal{M}(n+1)$. Since $\mathcal{M}(n+1)$ is recursively generated, we must also have

$$
Y^{n-1} \equiv Y^{n} X=a_{n} X^{n+1}+p_{n-1}(X) X+q_{n-1}(Y) X .
$$

If $a_{n}=0, \mathcal{S}_{n}(n-1) \bigcup\left\{X^{n}\right\}$ is linearly dependent, a contradiction. Thus, $a_{n} \neq$ 0 , which implies that $X^{n+1}$ can be written in terms of columns of lower degree. Moreover, from (4.4) we obtain $Y^{n+1}=a_{n} X^{n-1}+Y p_{n-1}(X)+Y q_{n-1}(Y)$, so $Y^{n+1}$ is also a linear combination of columns of lower degree. Finally, from $Y X=1$ and the recursiveness of $\mathcal{M}(n+1)$, we see that the intermediate columns $Y^{i} X^{j}(i+j=$ $n+1$, with $i, j \geq 1$ ) are all identical to columns corresponding to monomials of degree $n-1$. It follows that $\mathcal{M}(n+1)$ is flat, and is thus a flat extension of $\mathcal{M}(n)$.

Case IV. Here $\mathcal{S}_{n-1}(n) \bigcup\left\{X^{n}\right\}$ we consider the case when $\mathcal{S}_{n}(n)$ is linearly independent; the result follows directly from Proposition 2.14 (without using the given extension $\mathcal{M}(n+1)$ ). This completes the proof for $Y X=1$.

We now assume that $\mathcal{M}(n)$ is positive, recursively generated, $Y X=0$ in $\mathcal{C}_{\mathcal{M}(n)}$, and $\mathcal{M}(n)$ admits a positive, recursively generated extension $\mathcal{M}(n+1)$. We consider again the various cases of column dependence relations that we examined in the proof of Theorem 3.1] If the first dependence relation in $\mathcal{S}_{n}(n)$ occurs at $X^{k}(2 \leq$ $k \leq n)$, then Proposition 4.2 implies that $\mathcal{M}(n)$ has a flat extension $\mathcal{M}(n+1)$. If the first dependence relation occurs at $Y^{k}(2 \leq k \leq n)$, then, without recourse to the given extension $\mathcal{M}(n+1)$, Proposition 3.5 implies that $\mathcal{M}(n)$ admits a flat extension. In the remaining case, $\operatorname{rank} \mathcal{M}(n)=2 n+1$, so the result follows from Proposition 3.6 (again, without using the given extension $\mathcal{M}(n+1)$ ).

\section{Some Examples Illustrating Theorems 2.1 and 3.1}

Example 1.6] illustrates Case III of Theorem 2.1 We now present examples corresponding to other cases of Theorems 2.1 and 3.1 . 
Example 5.1. (Theorem 2.1 Case I) We illustrate $\mathcal{M}(3)$ in which $Y X=1$ and the first dependence relation in $\mathcal{S}_{3}(3)$ occurs at $X^{3}$. We define

$$
\mathcal{M}(3) \equiv \mathcal{M}(3)(\beta):=\left(\begin{array}{cccccccccc}
1 & 0 & 0 & a & 1 & a & 0 & 0 & 0 & 0 \\
0 & a & 1 & 0 & 0 & 0 & 2 a^{2} & a & 1 & a \\
0 & 1 & a & 0 & 0 & 0 & a & 1 & a & 2 a^{2} \\
a & 0 & 0 & 2 a^{2} & a & 1 & e & 0 & 0 & 0 \\
1 & 0 & 0 & a & 1 & a & 0 & 0 & 0 & 0 \\
a & 0 & 0 & 1 & a & 2 a^{2} & 0 & 0 & 0 & f \\
0 & 2 a^{2} & a & e & 0 & 0 & g & 2 a^{2} & a & 1 \\
0 & a & 1 & 0 & 0 & 0 & 2 a^{2} & a & 1 & a \\
0 & 1 & a & 0 & 0 & 0 & a & 1 & a & 2 a^{2} \\
0 & a & 2 a^{2} & 0 & 0 & f & a & a & 2 a^{2} & h
\end{array}\right),
$$

where $a>1$. Clearly $Y X=1$, and a calculation shows that $\operatorname{rank} \mathcal{M}(2)=5$. Thus, the set $\mathcal{B}:=\left\{1, X, Y, X^{2}, Y^{2}\right\}$ is linearly independent, and

$$
\left[X^{3}\right]_{\mathcal{B}}=-a e[1]_{\mathcal{B}}+\frac{a\left(2 a^{2}-1\right)}{a^{2}-1}[X]_{\mathcal{B}}-\frac{a^{2}}{a^{2}-1}[Y]_{\mathcal{B}}+\frac{a^{2} e}{2 a^{2}-1}\left[X^{2}\right]_{\mathcal{B}}+\frac{e\left(a^{2}-1\right)}{2 a^{2}-1}\left[Y^{2}\right]_{\mathcal{B}}
$$

We seek to impose the same relation in $\mathcal{C}_{\mathcal{M}(3)}$, which entails

$$
g=\frac{a\left(2 a^{2}-1\right)}{a^{2}-1} 2 a^{2}-\frac{a^{2}}{a^{2}-1} a+\frac{a^{2} e}{2 a^{2}-1} e=a^{2} \frac{8 a^{5}-10 a^{3}+3 a+a^{2} e^{2}-e^{2}}{\left(a^{2}-1\right)\left(2 a^{2}-1\right)}
$$

and

so that

$$
\frac{a\left(2 a^{2}-1\right)}{a^{2}-1} a-\frac{a^{2}}{a^{2}-1} 2 a^{2}+\frac{e\left(a^{2}-1\right)}{2 a^{2}-1} f=a,
$$

$$
f=a \frac{\left(a^{2}+a-1\right)\left(2 a^{2}-1\right)}{e\left(a^{2}-1\right)^{2}} .
$$

To compute $\mathcal{V}(\mathcal{M}(3))$, note that the column relation (5.1), together with $Y X=1$, gives rise to the equation

$$
x^{3}+a e-\frac{a\left(2 a^{2}-1\right)}{a^{2}-1} x+\frac{a^{2}}{a^{2}-1} \frac{1}{x}-\frac{a^{2} e}{2 a^{2}-1} x^{2}-\frac{e\left(a^{2}-1\right)}{2 a^{2}-1} \frac{1}{x^{2}}=0,
$$

or, equivalently,

$$
\begin{gathered}
\left(2 a^{2}-1\right)\left(a^{2}-1\right) x^{5}-a^{2} e\left(a^{2}-1\right) x^{4}-a\left(2 a^{2}-1\right)^{2} x^{3} \\
+a e\left(a^{2}-1\right)\left(2 a^{2}-1\right) x^{2}+a^{2}\left(2 a^{2}-1\right) x-e\left(a^{2}-1\right)^{2}=0 .
\end{gathered}
$$

Thus, $\mathcal{V}(\mathcal{M}(3))$ can have at most five points, so if $\operatorname{rank} \mathcal{M}(3) \leq \operatorname{card} \mathcal{V}(\mathcal{M}(3))$, then column $Y^{3}$ must be written in terms of the columns in $\mathcal{B}$ (in particular, $h$ is fully determined), and $\mathcal{M}(3)$ is therefore flat, in accordance with Proposition 2.5. For a specific numerical example, let $a:=2$ and $e:=1$, so that $f=\frac{70}{9}, g=\frac{740}{21}$, $h=\frac{2190400}{3087}$ and $Y^{3}=-\frac{1480}{21} \cdot 1-\frac{4}{3} X+\frac{14}{3} Y+\frac{740}{49} X^{2}+\frac{2960}{147} Y^{2}$. Then (5.2) becomes $21 x^{5}-12 x^{4}-98 x^{3}+42 x^{2}+28 x-9=0$, which has five real roots, as follows: $x_{1} \cong-2.0292, x_{2} \cong-0.521229, x_{3} \cong 0.282006, x_{4} \cong 0.658788$ and $x_{5} \cong 2.18106$. To calculate the densities we use Theorem 1.7. here $V$ is the $5 \times 5$ matrix whose entry in row $k$, column $\ell$ is $y_{\ell}^{i_{k}} x_{\ell}^{j_{k}}(1 \leq k, \ell \leq r)$, where $\left(i_{1}, j_{1}\right)=(0,0),\left(i_{2}, j_{2}\right)=(0,1)$, $\left(i_{3}, j_{3}\right)=(1,0),\left(i_{4}, j_{4}\right)=(0,2)$ and $\left(i_{5}, j_{5}\right)=(2,0)$. We then solve the equation $V \rho^{t}=\left(\beta_{i_{1}, j_{1}}, \ldots, \beta_{i_{5}, j_{5}}\right)^{t}$, where $\rho \equiv\left(\rho_{1}, \ldots, \rho_{5}\right)$ and $\left(\beta_{i_{1}, j_{1}}, \ldots, \beta_{i_{5}, j_{5}}\right)=(1,0,0,2,2)$. 
Thus, $\rho_{1} \cong 0.228429, \rho_{2} \cong 0.263185, \rho_{3} \cong 0.0174322, \rho_{4} \cong 0.31204, \rho_{5} \cong 0.178914$.

Example 5.2. (Theorem 2.1 Cases III and IV) Consider the moment matrix

$$
\mathcal{M}(3) \equiv \mathcal{M}(3)(\beta):=\left(\begin{array}{cccccccccc}
1 & 0 & 0 & 1 & 1 & 2 & 0 & 0 & 0 & 0 \\
0 & 1 & 1 & 0 & 0 & 0 & 3 & 1 & 1 & 2 \\
0 & 1 & 2 & 0 & 0 & 0 & 1 & 1 & 2 & 5 \\
1 & 0 & 0 & 3 & 1 & 1 & 0 & 0 & 0 & 0 \\
1 & 0 & 0 & 1 & 1 & 2 & 0 & 0 & 0 & 0 \\
2 & 0 & 0 & 1 & 2 & 5 & 0 & 0 & 0 & 3 \\
0 & 3 & 1 & 0 & 0 & 0 & 14 & 3 & 1 & 1 \\
0 & 1 & 1 & 0 & 0 & 0 & 3 & 1 & 1 & 2 \\
0 & 1 & 2 & 0 & 0 & 0 & 1 & 1 & 2 & 5 \\
0 & 2 & 5 & 0 & 0 & 3 & 1 & 2 & 5 & 33
\end{array}\right) .
$$

It is easy to see that $\mathcal{M}(3) \geq 0, Y X=1$ in $\mathcal{C}_{\mathcal{M}(3)}, \operatorname{rank} \mathcal{M}(3)=7$, and that $\mathcal{S}_{3}(3)$ is a basis for $\mathcal{C}_{\mathcal{M}(3)}$. The block $B(4)$ for a recursively generated extension is determined by the choice of $\beta_{07}=t$ and $\beta_{70}=u$. Following the proof of Proposition 2.14 we have $\operatorname{Ran} B(4) \subseteq \operatorname{Ran} \mathcal{M}(3)$, and a calculation of $\mathcal{M} \equiv[\mathcal{M}(3) ; B(4)]$ shows that $\mathcal{M}$ has the form of a moment matrix $\mathcal{M}(4)$ if and only if $(t+3)(u-150)=1$. For example, with $t=-2$ and $u=151$, we find the following column relations in $\mathcal{M}(4)$ that are not determined recursively from relations in $\mathcal{M}(3)$ :

$$
X^{4}=-20 \cdot 1+4 X+Y+9 X^{2}+7 Y^{2}-X^{3}-Y^{3}
$$

and

$$
Y^{4}=4 \cdot 1+9 X-20 Y-X^{2}+Y^{2}-X^{3}+7 Y^{3} .
$$

Together with $Y X=1$, these relations show that $\operatorname{card} \mathcal{V}(\mathcal{M}(4))=\operatorname{rank} \mathcal{M}(4)=$ 7 ; the $x$-coordinates of the points in $\left\{\left(x_{i}, \frac{1}{x_{i}}\right)\right\}_{i=1}^{7} \equiv \mathcal{V}(\mathcal{M}(4))$ are as follows: $x_{1} \cong-2.82238, x_{2} \cong-1.87650, x_{3} \cong-0.64947, x_{4} \cong 0.14873, x_{5} \cong 0.66898$, $x_{6} \cong 1.32445$ and $x_{7} \cong 2.20619$. Since $\mathcal{S}_{3}(3)$ is a basis for $\mathcal{C}_{\mathcal{M}(3)}$, Theorem 1.7 now implies that the densities $\left\{\rho_{i}\right\}_{i=1}^{7}$ of the unique representing measure $\mu=$ $\sum_{i=1}^{7} \rho_{i} \delta_{\left(x_{i}, \frac{1}{x_{i}}\right)}$ for $\mathcal{M}(4)$ are as follows: $\rho_{1} \cong 0.00754, \rho_{2} \cong 0.07579, \rho_{3} \cong 0.41491$, $\rho_{4} \cong 0.00025, \rho_{5} \cong 0.43154, \rho_{6} \cong 0.01146$ and $\rho_{7} \cong 0.05851$.

We can illustrate the second part of Case IV and also Case III if we use $t=-3$ and $u=150$, so that $M$ is not a moment matrix. In this case, the $C$ block of $M$ satisfies $C_{11}=79$. Following the proof of Proposition 2.14 we redefine $C_{11}:=80, C_{15}:=1$, and we compute $C_{55}\left(=\beta_{80}\right)=1036$, so as to define a positive rank 8 moment matrix $M(4)$ in which the first dependence relation in $S_{4}(4)$ is of the form $Y^{4}=-16 \cdot 1+18 X-21 Y+8 X^{2}+8 Y^{2}-3 X^{3}+6 Y^{3}-X^{4}$ (i.e., $M(4)$ is in Case III). This relation and $Y X=1$ determine $\mathcal{V} \equiv \mathcal{V}(\mathcal{M}(4)$ ), and a calculation shows that $\operatorname{card} \mathcal{V}=\operatorname{rank} \mathcal{M}(4)=8$; the $x$-coordinates of the points $\left\{\left(x_{i}, \frac{1}{x_{i}}\right)\right\}_{i=1}^{8} \equiv \mathcal{V}$ are as follows: $x_{1} \cong-3.63582, x_{2} \cong-2.02578, x_{3} \cong$ $-0.840968, x_{4} \cong-0.644996, x_{5} \cong 0.149637, x_{6} \cong 0.670287, x_{7} \cong 1.14158$ and $x_{8} \cong 2.18606$. Since the unique representing measure $\mu$ for the flat extension $M(5)$ of $M(4)$ (guaranteed by Proposition 2.7) satisfies card $\operatorname{supp} \mu=\operatorname{rank} M(4)=8$, it follows that $\operatorname{supp} \mu=\mathcal{V}$ and that the densities of $\mu$ (computed as in Theorem 1.7) are $\rho_{1} \cong 0.00087, \rho_{2} \cong 0.07462, \rho_{3} \cong 0.02883, \rho_{4} \cong 0.39396, \rho_{5} \cong 0.00025$, $\rho_{6} \cong 0.43357, \rho_{7} \cong 0.00612$ and $\rho_{8} \cong 0.06177$.

We next turn to examples illustrating Theorem 3.1 
Example 5.3. (cf. Proposition 3.4) We illustrate $\mathcal{M}(2)$ with $Y X=0$, where the first dependence relation in $\mathcal{S}_{2}(2)$ occurs at $X^{2}$. Consider the moment matrix

$$
\mathcal{M}(2):=\left(\begin{array}{cccccc}
1 & a & 0 & b & 0 & d \\
a & b & 0 & e & 0 & 0 \\
0 & 0 & d & 0 & 0 & g \\
b & e & 0 & f & 0 & 0 \\
0 & 0 & 0 & 0 & 0 & 0 \\
d & 0 & g & 0 & 0 & h
\end{array}\right) .
$$

A straightforward calculation reveals that with $b>a^{2}, d>0, e=\frac{b^{2}}{a}, f=\frac{b^{3}}{a^{2}}$ and $h>\frac{b d^{3}-a^{2} g^{2}+b g^{2}}{d\left(b-a^{2}\right)}, \mathcal{M}(2) \geq 0, \operatorname{rank} \mathcal{M}(2)=4, Y X=0$ in $\mathcal{C}_{\mathcal{M}(2)}$, and $X^{2}=\frac{b}{a} X$. The variety $\mathcal{V}(\mathcal{M}(2))$ associated to this matrix consists of the intersection of $y x=0$ with $x\left(x-\frac{b}{a}\right)=0$, so card $\mathcal{V}(\mathcal{M}(2))=\infty$. Now, in any recursively generated extension $\mathcal{M}(3)$ we must have $X^{3}=\frac{b}{a} X^{2}=\frac{b^{2}}{a^{2}} X$. Also, the form of a block $B(3)$ is

$$
B(3) \equiv\left(\begin{array}{cccc}
e & 0 & 0 & g \\
f & 0 & 0 & 0 \\
0 & 0 & 0 & h \\
p & 0 & 0 & 0 \\
0 & 0 & 0 & 0 \\
0 & 0 & 0 & q
\end{array}\right)
$$

where $p:=\frac{b^{4}}{a^{3}}$ and $q$ is free. Following the proof of Proposition 3.4 the existence of a flat extension $\mathcal{M}(3)$ depends on the verification of (3.9), that is, $\left[X^{3}\right]_{\{1,2,3,6\}} \mathcal{M}(2)_{\{1,2,3,6\}}^{-1}\left[Y^{3}\right]_{\{1,2,3,6\}}=0 . \quad$ A straightforward calculation shows that this is indeed the case, so $\mathcal{M}(2)$ admits a flat extension.

Example 5.4. (cf. Proposition 3.6 and [CuFi6, Example 5.6]) Consider the moment matrix

$$
\mathcal{M}(2):=\left(\begin{array}{cccccc}
1 & 1 & 1 & 2 & 0 & 3 \\
1 & 2 & 0 & 4 & 0 & 0 \\
1 & 0 & 3 & 0 & 0 & 9 \\
2 & 4 & 0 & 9 & 0 & 0 \\
0 & 0 & 0 & 0 & 0 & 0 \\
3 & 0 & 9 & 0 & 0 & 28
\end{array}\right)
$$

Observe that $\operatorname{rank} \mathcal{M}(2)=5$, and that $Y X=0$ in $\mathcal{C}_{\mathcal{M}(2)}$, so that $\mathcal{S}_{2}(2)$ is a basis for $\mathcal{C}_{\mathcal{M}(2)}$. A block $B(3)$ for a recursively generated extension is completely determined by the choice of $\beta_{05}=p$ and $\beta_{50}=q$. With these choices, the $C$ block in the flat extension $[\mathcal{M}(2) ; B(3)]$ has entries $C_{11}=(p-18)^{2}+42, C_{14}=C_{41}=1$, and $C_{44}=(q-84)^{2}+262$. It is then clear that $[\mathcal{M}(2) ; B(3)]$ is not a moment matrix, so $\beta^{(4)}$ has no 5 -atomic representing measure. To construct a 6 -atomic representing measure, we modify the two key entries, $C_{11}$ and $C_{14}$, as dictated by the proof of Proposition 3.6 By taking $p=18, q=84, u=43$, it is easy to see that $\operatorname{rank} \mathcal{M}(3)=1+\operatorname{rank} \mathcal{M}(2)(=6)$ precisely when $v=263$. As in the proof of Proposition 3.6 we claim that $\mathcal{M}(3)$ admits a flat extension $\mathcal{M}(4)$. We first exhibit a unique block $B(4)$, subordinate to $Y X=0$, such that Ran $B(4) \subseteq$ Ran $\mathcal{M}(3)$. In any such block, $Y X^{3}=Y^{2} X^{2}=Y^{3} X=0$. In $Y^{4}$, all the entries are determined from $\mathcal{M}(3)$ and $Y X=0$, except $s:=\left\langle Y^{4}, Y^{3}\right\rangle$. Now, in $\mathcal{C}_{\mathcal{M}(3)}$ we 
have a dependence relation

$$
Y^{3}=-5 \cdot 1+7 X+11 Y-X^{3}
$$

since a flat extension must necessarily be recursively generated, in $\mathcal{C}_{\mathcal{M}(4)}$ we must have

$$
Y^{4}=-5 Y+11 Y^{2}
$$

whence $s=\left\langle-5 Y+11 Y^{2}, Y^{3}\right\rangle=-5 \beta_{4,0}+11 \beta_{5,0}=784$. Also, in $X^{4}$, all values are determined except $r:=\left\langle X^{4}, X^{3}\right\rangle$; following Proposition 3.6] we claim that there is a unique value for $r$ such that $X^{4} \in \operatorname{Ran} \mathcal{M}(3)$. For, relative to the linearly independent set of columns $\left\{1, X, Y, X^{2}, Y^{2}, X^{3}\right\}$ in $\mathcal{C}_{\mathcal{M}(3)}$,

$$
X^{4}=(3 r-243) \cdot 1+(481-6 r) X+(81-r) Y+7 X^{2}+(r-81) X^{3},
$$

and since $\left\langle X^{4}, Y^{3}\right\rangle$ must be zero, we obtain

$$
\begin{aligned}
0 & =\left\langle(3 r-243) \cdot 1+(481-6 r) X+(81-r) Y+7 X^{2}+(r-81) X^{3}, Y^{3}\right\rangle \\
& =(3 r-243) \beta_{3,0}+(81-r) \beta_{4,0}=(3 r-243) \cdot 9+(81-r) \cdot 28=81-r,
\end{aligned}
$$

whence $r=81$. The unique 6 -atomic representing measure associated with $\mathcal{M}(3)$ is then given by $\left(x_{1}, y_{1}\right) \cong(2.16601,0),\left(x_{2}, y_{2}\right) \cong(0.782816,0),\left(x_{3}, y_{3}\right) \cong$ $(-2.94883,0),\left(x_{4}, y_{4}\right) \cong(0,0.463604),\left(x_{5}, y_{5}\right) \cong(0,3.06043),\left(x_{6}, y_{6}\right) \cong$ $(0,-3.52404), \rho_{1} \cong 0.393081, \rho_{2} \cong 0.203329, \rho_{3} \cong 0.00359018, \rho_{4} \cong 0.0821253$, $\rho_{5} \cong 0.316218, \rho_{6} \cong 0.00165656$.

\section{An Application to the Full Moment Problem}

We conclude with a new proof of Theorem 1.3 We require the following preliminary result, which was given in [Fia3] for complex moment matrices, but also holds for $\mathcal{M}(\infty)(\beta)$.

Lemma 6.1. (Fia3 Proposition 4.2]) Let $\beta \equiv \beta^{(\infty)}$ be a full sequence such that $\mathcal{M}(\infty) \geq 0$. Then $\mathcal{M}(n)$ is positive and recursively generated for each $n \geq 1$.

Theorem 6.2. (cf. [Sto1]) Let $P \in \mathbb{R}[x, y]$ with $\operatorname{deg} P \leq 2$. The sequence $\beta \equiv \beta^{(\infty)}$ has a representing measure supported in $P(x, y)=0$ if and only if $\mathcal{M} \equiv \mathcal{M}(\infty)(\beta) \geq 0$ and $P(X, Y)=0$ in $\mathcal{C}_{\mathcal{M}}$.

Proof. Let $\mu$ be a representing measure for $\beta$ supported in $\mathcal{Z}(P)$. For each $n \geq 2$, $\mu$ is a representing measure for $\beta^{(2 n)}$, so (1.4) and (1.5) imply that $\mathcal{M}(n)(\beta) \geq 0$ and $P(X, Y)=0$ in $\mathcal{C}_{\mathcal{M}(n)}$; thus $\mathcal{M} \geq 0$ and $P(X, Y)=0$ in $\mathcal{C}_{\mathcal{M}}$.

For the converse, since $\mathcal{M} \geq 0$, Lemma 6.1 implies that for $n \geq 2, \mathcal{M}(n+1)$ is positive and recursively generated, so $\mathcal{M}(n)$ has a positive, recursively generated extension. Since $P(X, Y)=0$ in $\mathcal{C}_{\mathcal{M}}$, the same is true in $\mathcal{C}_{\mathcal{M}(n)}$. Suppose now that $P(x, y)=0$ is a nondegenerate hyperbola. Let $\Phi$ be an injective degree-one mapping of the plane onto itself such that $\Phi(\mathcal{Z}(P))=\{(x, y): y x=1\}$ (cf. Section (1), and let $\tilde{\beta}^{(2 n)}$ be the sequence corresponding to $\beta^{(2 n)}$ via Proposition 1.9. Proposition 1.9(ix) implies that $\tilde{\mathcal{M}}(n) \equiv \mathcal{M}(n)(\tilde{\beta})$ has a positive, recursively generated extension $\tilde{\mathcal{M}}(n+1)$, and Proposition 1.9 (vi) implies that $Y X=1$ in $\mathcal{C}_{\tilde{\mathcal{M}}(n)}$. Theorem 1.5(iv) now implies that $\tilde{\beta}^{(2 n)}$ has a representing measure supported in $y x=1$, so Proposition [1.9)(v) implies that $\beta^{(2 n)}$ has a representing measure supported in $P(x, y)=0$; the existence of a representing measure for $\beta$ supported in $P(x, y)=0$ now follows from Theorem 1.4 
The cases of other conics are handled similarly. For degenerate hyperbolas, we use the case of Theorem 1.1 for $y x=0$. For parabolas and ellipses we use CuFi7, Theorem 1.4] and CuFi5. Theorem 3.5], respectively. The cases of lines is treated directly in CuFi2, Theorem 2.1].

Remark 6.3. (i) By analogy with properties $(A)$ and $\left(A_{n}^{\prime}\right)$ (cf. Section 1), consider also the following possible properties for a polynomial $P \in \mathbb{R}[x, y]$ :

$$
\beta \equiv \beta^{(2 n)} \text { has a representing measure supported in } \mathcal{Z}(P) \text { if and only if }
$$

$\left(A_{n}\right) \quad \mathcal{M}(n)(\beta)$ is positive semi-definite, recursively generated

$$
\text { and } P(X, Y)=0 \text { in } \mathcal{C}_{\mathcal{M}(n)} \text {. }
$$

$\beta \equiv \beta^{(\infty)}$ has a representing measure supported in $\mathcal{Z}(P)$ if and only if

$$
\begin{gathered}
\mathcal{M}(\infty)(\beta) \text { is positive semi-definite, } P(X, Y)=0 \text { in } \mathcal{C}_{\mathcal{M}(\infty)}, \\
\text { and } \operatorname{rank} \mathcal{M}(\infty) \leq \operatorname{card} \mathcal{V}(\mathcal{M}(\infty)) .
\end{gathered}
$$

Theorem 1.4 readily implies the following implications concerning possible properties enjoyed by a given polynomial $P$ :

$$
\begin{array}{clc}
\left(A_{n}\right) \text { holds for all } n \geq \operatorname{deg} P & \Rightarrow & \left(A_{n}^{\prime}\right) \text { holds for all } n \geq \operatorname{deg} P \\
\Downarrow & & \Downarrow \\
(A) \text { holds } & \Rightarrow & \left(A^{\prime}\right) \text { holds. }
\end{array}
$$

First-degree polynomials $P$ satisfy $\left(A_{n}\right)$ for all $n \geq \operatorname{deg} P$, but our results show that second-degree polynomials do not. However, second-degree polynomials satisfy $\left(A_{n}^{\prime}\right)$ for all $n \geq \operatorname{deg} P$, and also $(A)$. Stochel Sto1 has identified cubics which fail to satisfy $(A)$, but we know of no example of a polynomial $P$ that fails to satisfy $\left(A^{\prime}\right)$, or $\left(A_{n}^{\prime}\right)$ for some $n \geq \operatorname{deg} P$.

(ii) The full moment problem on compact semi-algebraic sets in $\mathbb{R}^{n}$ was solved by K. Schmüdgen Schm1. Recently, the analysis of the semi-algebraic case was extended to non-compact sets by V. Powers and C. Scheiderer PoSc] (cf. KuMa, Sche, Schm2]).

\section{REFERENCES}

[AhKr] N.I. Ahiezer and M. Krein, Some Questions in the Theory of Moments, Transl. Math. Monographs, vol. 2, American Math. Soc., Providence, 1962.

[Akh] N.I. Akhiezer, The Classical Moment Problem, Hafner Publ. Co., New York, 1965.

[Atk] K. Atkinson, Introduction to Numerical Analysis, Wiley and Sons, 2nd. Ed. 1989.

[CuFi1] R. Curto and L. Fialkow, Solution of the truncated complex moment problem with flat data, Memoirs Amer. Math. Soc. no. 568, Amer. Math. Soc., Providence, 1996.

[CuFi2] R. Curto and L. Fialkow, Flat extensions of positive moment matrices: Relations in analytic or conjugate terms, Operator Th.: Adv. Appl. 104(1998), 59-82.

[CuFi3] R. Curto and L. Fialkow, Flat extensions of positive moment matrices: Recursively generated relations, Memoirs Amer. Math. Soc. no. 648, Amer. Math. Soc., Providence, 1998.

[CuFi4] R. Curto and L. Fialkow, The truncated complex $K$-moment problem, Trans. Amer. Math. Soc. 352(2000), 2825-2855.

[CuFi5] R. Curto ad L. Fialkow, The quadratic moment problem for the unit disk and unit circle, Integral Equations Operator Theory 38(2000), 377-409.

[CuFi6] R. Curto and L. Fialkow, Solution of the singular quartic moment problem, J. Operator Theory 48(2002), 315-354.

[CuFi7] R. Curto and L. Fialkow, Solution of the truncated parabolic moment problem, Integral Equations Operator Theory, to appear. 
[CuFi8] R. Curto and L. Fialkow, Truncated $K$-moment problems in several variables, J. Operator Theory, to appear.

[Dou] R.G. Douglas, On majorization, factorization, and range inclusion of operators on Hilbert spaces, Proc. Amer. Math. Soc. 17(1966), 413-415.

[Fia1] L. Fialkow, Positivity, extensions and the truncated complex moment problem, Contemporary Math. 185(1995), 133-150.

[Fia2] L. Fialkow, Minimal representing measures arising from rank-increasing moment matrix extensions, J. Operator Theory 42(1999), 425-436.

[Fia3] L. Fialkow, Truncated complex moment problems with a $Z \bar{Z}$ relation, Integral Equations Operator Theory 45(2003), 405-435.

[FiPe] L. Fialkow and S. Petrovic, A moment matrix approach to multivariable cubature, Integral Equations Operator Theory, to appear.

[KrNu] M.G. Krein and A.A. Nudel'man, The Markov Moment Problem and Extremal Problems, Transl. Math. Monographs, vol. 50, American Mathematical Society, Providence, R.I., 1977.

[KuMa] S. Kuhlmann and M. Marshall, Positivity, sums of squares and the multidimensional moment problem, Trans. Amer. Math. Soc. 354(2002), 4285-4301.

[PoSc] V. Powers and C. Scheiderer, The moment problem for non-compact semialgebraic sets, Advances in Geometry 1(2001), 71-88.

[PuVa] M. Putinar and F.-H. Vasilescu, Solving moment problems by dimensional extension, Ann. of Math. (2) 149(1999), no. 3, 1087-1107.

[Sche] C. Scheiderer, Sums of squares of regular functions on real algebraic varieties, Trans. Amer. Math. Soc. 352(2000), 1039-1069.

[Schm1] K. Schmüdgen, The K-moment problem for semi-algebraic sets, Math. Ann. 289(1991), 203-206.

[Schm2] K. Schmüdgen, On the moment problem of closed semi-algebraic sets, J. Reine Angew. Math. 558 (2003), 225-234.

[ShTa] J.A. Shohat and J.D. Tamarkin, The Problem of Moments, Math. Surveys I, American Math. Soc., Providence, 1943.

[Smu] J.L. Smul'jan, An operator Hellinger integral (Russian), Mat. Sb. 91(1959), 381-430.

[Sto1] J. Stochel, Moment functions on real algebraic sets, Ark. Mat. 30(1992), 133-148.

[Sto2] J. Stochel, Solving the truncated moment problem solves the moment problem, Glasgow J. Math. 43(2001), 335-341.

[StSz1] J. Stochel and F.H. Szafraniec, Algebraic operators and moments on algebraic sets, Portugal. Math. 51(1994), 25-45.

[StSz2] J. Stochel and F.H. Szafraniec, The complex moment problem and subnormality: A polar decomposition approach, J. Funct. Anal. 159(1998), 432-491.

[Wol] Wolfram Research, Inc., Mathematica, Version 4.2, Wolfram Research, Inc., Champaign, IL, 2002.

Department of Mathematics

The University of Iowa

IOWA CiTy, IA 52242-1419

USA

E-mail address: rcurto@math.uiowa.edu

Department of Computer Science

State University of New York

New Paltz, NY 12561

USA

E-mail address: fialkowl@newpaltz.edu 